\title{
Investigation of De Speckling Techniques for Echocardiographic Images:
}

\section{A Review}

\author{
Rehan Ahmad ${ }^{1}$, Dr. Mohan Awasthy² \\ 1 Mr. Rehan Ahmad, is the faculty at NMIMS, Mukesh Patel School of Technology Management and Engineering, \\ Shirpur. Rehan.ahmad@nmims.edu, electron3011@gmail.com \\ 2 Mohan Awasthy, Ph.D. is the faculty at NMIMS, Mukesh Patel School of Technology Management and \\ Engineering, Shirpur. Mohan.Awasthy@nmims.edu
}

\begin{abstract}
:
Speckle noise corrupt the major part of ultrasound image, because of which the quality deteriorate and loss of valuable information leads to false diagnosis. A large community of images like synthetic aperture radar (SAR) image, Synthetic image, and simulated ultrasound image, require despeckling at pre-processing stage for better processing. Cleaning the speckle from image and preserving the edge details is a vital task. Nowadays not only despeckling is considered as an important process but also preserving information at boundary and edges of image is also important. As most of the algorithms able to remove speckle noise but fails to preserve the details of edges. This paper covers several recent methods for removal of speckle noise along with various metrics opted for comparisons. The distinctive part of this paper is, a mathematical and parametric review has been done. Also a table is also included which summarizes the entire paper.
\end{abstract}

Keywords: Ultrasound image, Speckle noise, Wiener filter, Average filter, Wavelet filter, Adaptive filter, Fractional filter. 


\section{Introduction:}

Medical imaging is sensitive to mechanism of imaging, object being imaged, instruments involved, reconstruction through imaging algorithms, and optical display. Medical imaging is a way of collecting information about an organ or tissue with the help of predefined characteristics collectively displayed in the form of image [1]. Sound waves (also in the form of acoustic wave, commonly also known as ultrasound waves) used as a diagnostic modality to identify characteristics of tissue(s), measurement of flow of blood, and display of structure of anatomy. Merits of ultrasound include non-invasive, real time processing of image, safety, specialized contrast agents, economical cost of operation, faster processing, non-ionizing radiation, unique temporal resolution and portability. In medical domain the preferable wavelength of ultrasound wave is $2 \mathrm{MHz}$ to $10 \mathrm{MHz}$, out of this $2 \mathrm{MHzto} 5 \mathrm{MHz}$ is most commonly used frequency band for imaging. The use of ultrasound defined its range of frequency e.g. therapeutic ultrasound uses $0.8 \mathrm{MHz}-8 \mathrm{MHz}$, diagnostic ultrasound $1 \mathrm{MHz}-$ $40 \mathrm{MHz}$, acoustic microscopy upto $2 \mathrm{GHz}$ [2].

Suppose a transducer emits an acoustic signal of intensity $s(x, y)$ with a pulse $w(t)$ transmitted through a medium having attenuation coefficient $\mu$, reflected by a tissue (of biological property) of reflectivity $R(x, y, z)$, tissue is at a distance $z$ from the transducer[1]. The measured intensity of time varying acoustic signal $\mathrm{J}_{\mathrm{r}}(\mathrm{t})$ across the region $R$ can be expressed as-

$\mathrm{J}_{\mathrm{r}}(\mathrm{t})=\mathrm{K}\left|\iiint R\left(e^{-2 \mu z} / z\right) R(x, y, z) s(x, y) \widetilde{\mathrm{w}}(t-2 z / c) d x d y d z\right|$

Where $\mathrm{K}$ represents normalizing constants, $\bar{\omega} t$ represents received pulse, c represents velocity of acoustic signal in the medium.

The reflected signal from the tissue when recorded represented as

$\mathrm{J}_{\mathrm{c}} \mathrm{r}(\mathrm{t})=\mathrm{K}\left|\iiint R(x, y, z) s(x, y) \overline{\mathrm{w}}(t-2 z / c) d x d y d z\right|$

For clinical use, medical imaging has offered various techniques for diagnosis of shape, size, and structure of organ. Among available various techniques ultrasound imaging is commonly used in several sections of human anatomy for diagnosis like pancreas, kidneys, gallbladder, neck, chest, liver etc. The only demerit of ultrasound image (USG image) is poor quality because of presence of speckle noise. All USG images are affected by two types of consistent obstructions, constructive and destructive obstructions of backscattered echoes from randomly distributed scattered energy, and commonly known as speckle. Speckle noise is a multiplicative type which attenuates the precious information of object being imaged [3]. In presence of speckle noise results of various image processing applications such as image feature extraction, image classification, and image segmentation are hampered. Also edge detection, boundary 
enhancement between organs are few modern day challenges in medical image processing domain. It has been recorded by several researchers that, while dealing (reducing or removing) speckle noise it may kill valuable features of ultrasound images. It is suggested by researchers, despeckling algorithms should be designed in such a way that minimum (or no loss of) information loss, and clearing maximum speckling noise. Despeckling process in USG images can be categorized as image averaging, image filtering [3]. Image averaging can be defined as taking average of interrelated arrangements of ultrasound in frequency or spatial domain. These interrelated sampled images can be sampled at distinct views, distinct frequency interval and distinct time interval. Because of which loss in resolution takes place. Filtering methods can be subcategorized as single scale spatial filtering and multiscale spatial filtering.

Single scale spatial filtering having subsets like non-linear noise cleaning and linear noise cleaning [4]. Linear noise cleaning is further segmented as spatial domain filtering, homomorphic filtering and Fourier domain processing. Non-linear noise cleaning has subsets like outlier noise cleaning technique, Median filter, Pseudomedian filter, wavelet denoising [4][5]. Multiscale spatial filtering can be classified in different categories such as pyramid [6], curvelet [7], ridgelet [8][9], wavelet [10].

Nowadays many computer aided diagnosis systems which processes ultrasound images are used for prediction of a disease. A most commonly used Bmode grayscale ultrasound is used in clinical application because of its simplicity and ease of operation [11]. But major demerit of ultrasound is, it is greatly affected by speckle noise at the time of USG image capturing, which results into poor quality of image, which leads to error in diagnosis [12][13].

The major goal of despeckling process is preserving the original image data in order to enhance the diagnosis accuracy. A generalized model of speckle noise is

$\mathrm{w}(\mathrm{x}, \mathrm{y}) \sim \mathrm{u}(\mathrm{x}, \mathrm{y}) \mathrm{v}(\mathrm{x}, \mathrm{y})+\xi(\mathrm{x}, \mathrm{y})$

Where, $w(x, y)$ represents observed image, $u(x, y)$ represents original image, $v(x, y)$ represents multiplicative components of speckle noise, $\xi(x, y)$ represents additive components of speckle noise.

Followings are research papers which describes the various despeckling methods exercised for USG images-

A high computational efficiency is required, if a wiener filter is used in frequency domain. To overcome this issue an enhanced wiener filter was proposed and implemented by Fabio Baselice et. al. [14][2017]. This filter has a distinctive characteristic of combining the 
edges with preserving details, and decrement in noise, all stated parameters are locally adapted by the filter. The multiplicative model of a USG scanner is

$$
A(i, j)=B(i, j) C(i, j)
$$

$A(i, j)$ represents the amplitude signal, $B(i, j)$ represents original signal, $C(i, j)$ speckle noise $(i, j)$ represents space indexes of acquisition geometry.

A homomorphic filtering transformation provides an additive model from multiplicative model. By applying a log transform, the acquisition model turn into

$$
\begin{aligned}
A^{\prime}(i, j) & =|\log A(i, j)|=\log [B(i, j]+\log [C(i, j)] \\
& =B^{\prime}(i, j)+C^{\prime}(i, j)
\end{aligned}
$$

When a wiener filter is applied to an acquisition model, can be defined as

$$
\operatorname{Hw}(i, j)=\frac{P_{y^{\prime}}(\zeta, \eta)}{P_{y^{\prime}}(\zeta, \eta)+\alpha W_{n^{\prime}}(\zeta, \eta)}
$$

Where

$\mathrm{P}_{\mathrm{y}^{\prime}}(\zeta, \eta)$ power spectrum of noise free signal with $\mathrm{B}^{\prime}(\mathrm{i}, \mathrm{j})$ with $(\zeta, \eta)$ 2dimensional spatial frequencies.

$\alpha$ is a scalar value used for tuning filter intensity. For standard wiener filter it is 1 .

An image model based on Markov Random Field (MRF) was constructed for adding external information on image. A Gaussian MRF model was used to define the relation between pixel under process and neighborhood pixels. A hyper parameter is a distillation in G-MRF and is called Local Gaussian- MRF (LGMRF), is a link which defines the relationship between each pixels and adjoining pixels. A high value of hyper parameter indicates weak relationship strength or vice versa. The hyper parameters of LGMRF can be forecast using below equation-

$$
\sigma_{\mathrm{p}, \mathrm{q}}=\sqrt{\frac{\left(y_{p}^{\prime}-y_{q}{ }^{\prime}\right)^{2}}{9}}
$$

Where p,q are neighboring pixels, y'p and y'q are the values of pixel under process. For each pair of pixels value of $\sigma$ can be determined.

The maneuver of hyper parameter is for adjustment of filter intensity to restore edges and other information during noise elimination process. Suppose pixels $\mathrm{p}, \mathrm{q}$ share hyper parameter $\sigma_{\mathrm{p}, \mathrm{q}}$, chances are high that these pixels possess various values, so that wiener filter should not have the 
solution. The value of $\alpha$ and hyper parameter $\sigma$ could be used for construction of hyper parameter map (HP map). The proposed method as depicted in below figure-

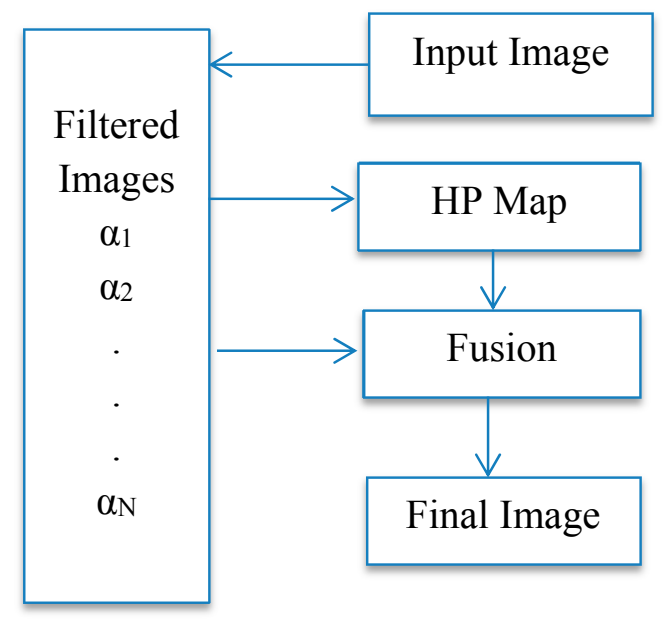

Fig. 1. Functioning diagram of Enhanced Wiener filter

The homo morphically transformed input image is filtered $\mathrm{N}$ times through the Weiner kernel of eq. (3) using the coefficients $\alpha_{1}, \alpha_{2}, \ldots, \alpha_{\mathrm{N}}$ and selected according to

$$
\alpha_{\mathrm{n}}=\left(\frac{n}{10}\right)^{\beta}
$$

$\mathrm{n}=1, \ldots, \mathrm{N}$.

$\mathrm{N}$ number of images with various intensity and with a specific regularization level. The performance of all these $\mathrm{N}$ filtered images are estimated using equation (4) for hyper parameter. Every regularized image will creates a hyper parameter map. The global map can be calculated by averaging the maps, prejudice can be avoided by performing on N/2 images. The global hyper parameter map was used for merging to have one final image. For each pixel $p$ out of $\mathrm{N}$ solutions, the best fir solution is selected based upon hyper parameter value. This process provides optimum solution for each pixel.

The suggested methodology offers great proficiency at minimum supervision.

An approach based upon radiation intensity of pixel and average filtering was proposed by Alex F. de Araujo et. al. [15] [2016], this method identify pixels lies on the boundary of region of interest, reducing the intensity of pixels interest and increasing the intensity of rest of the pixels. The intensity of radiation of a pixel can be defined as

$$
\mathrm{r}=\mathrm{p} / \mathrm{a}
$$


where circumference of region covered by radiation, $r$ represents the strength of radiation and $p$ represents power. Suppose the radiation is covering an area of circular in shape, $\mathrm{m}$ indicate a pixel whose gray level can be

$$
\mathrm{r}_{\mathrm{m}}=\mathrm{Im}_{\mathrm{m} / 4 \mathrm{r}^{2}}
$$

$\mathrm{r}$ represents the perimeter affected by radiation of pixel $\mathrm{m}$. To calculate the chances of a pixel to be noisy, the radiation intensity of pixel $\mathrm{m}$ can be estimated by summing up the neighboring pixels and considering the parameter Ji estimated for each pixel I as below-

$$
\mathrm{r}_{\mathrm{m}}=\sum_{m=1}^{w / 2}\left[\left(\frac{\mathrm{Im}}{4 \Pi \mathrm{r} 2}\right)-\mathrm{Ji}\right]
$$

$\mathrm{w}$ represents the width of initial image and $\mathrm{w} / 2$ represents radius of pixel indicated in circular format. If a pixel is affected by the radiation effect of neighboring pixel, chances are high that pixel belongs to a homogeneous region or being noisy. The flowchart below depicts the sequence of a newly proposed smoothing filter

This method relies on selective average filter for noise removal and adaptively defines the $3 \times 3$ size of filter window, to accumulate the radiation of pixel under process and surrounding pixels. At first stage the smoothing provides are of low intensity to ward off the loss of important information. To skip the enormous change in gray level of picture elements, parameter $\mathrm{T}_{\mathrm{i}}$ added in eq. (3), prevents steep change with reference to original value. This parameter for pixels a and $\mathrm{b}$ can be written as-

$$
\mathrm{T}_{\mathrm{i}}=\mathrm{I}_{\mathrm{a}}-\mathrm{I}_{\mathrm{b}}
$$

$\mathrm{I}_{\mathrm{a}}$ represents original gray value of picture element, and $\mathrm{I}_{\mathrm{b}}$ represents the gray value of emitting picture element. The radiation of each picture element ' $a$ ' can be estimated using eq. (3), computing the probability of picture element whether affected by noise. Using larger convolution window the magnitude of each affected picture element can be computed, and the rest of picture elements can be recomputed using average filter with decrepit smoothing effect using smaller convolution window. 
The results of proposed method and other methods are shown below-
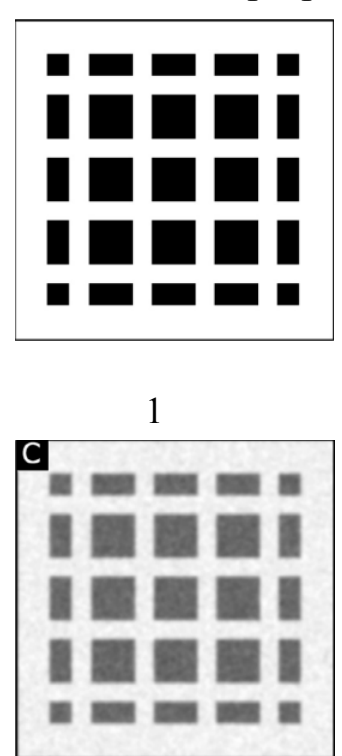

5

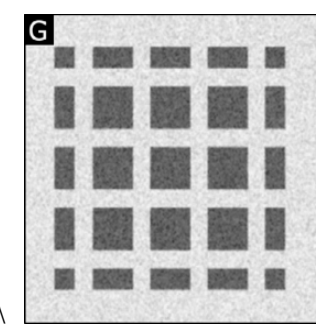

9
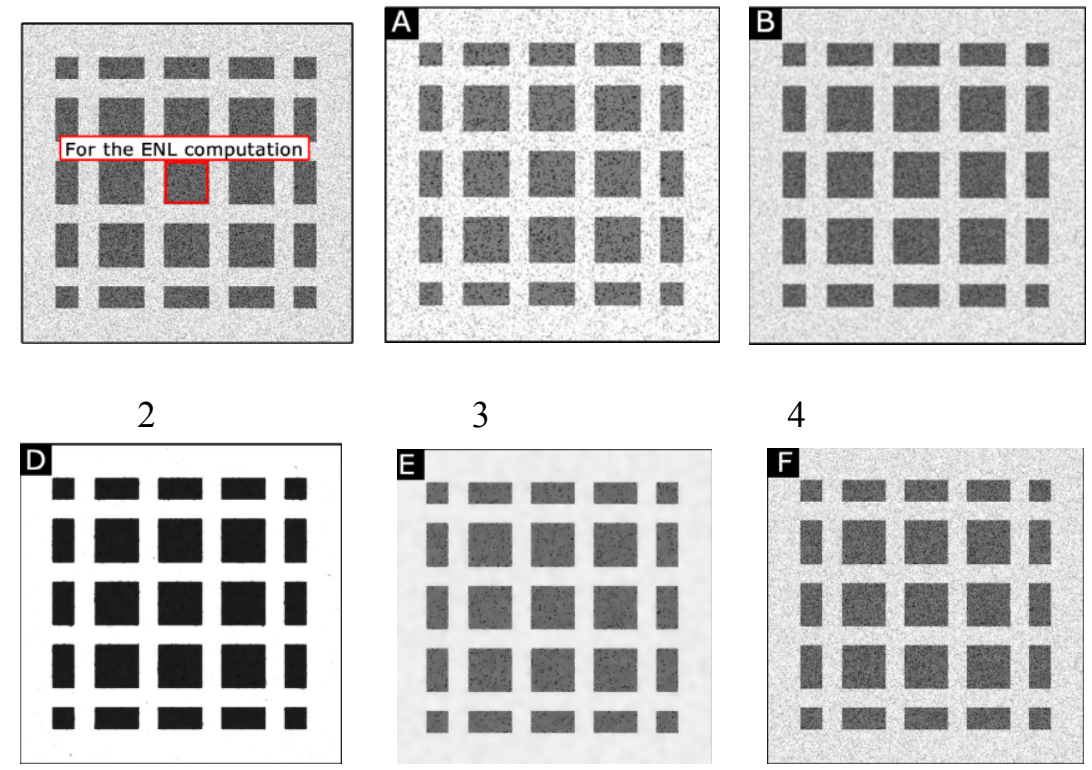

6

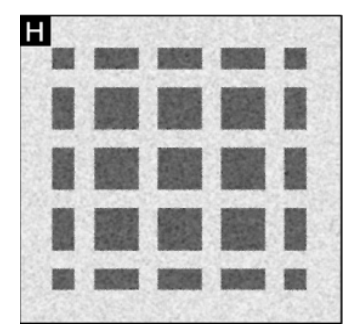

10

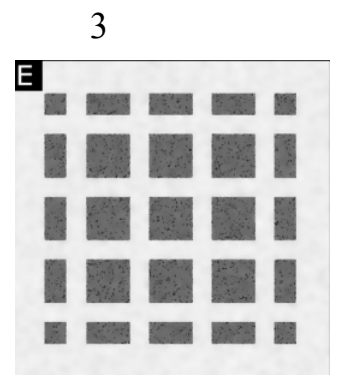

7

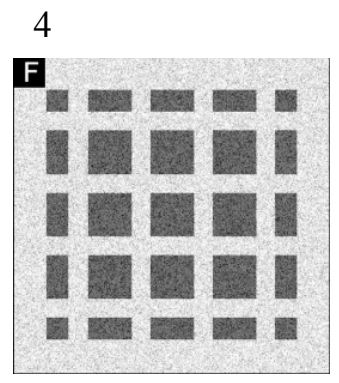

8

Fig. 1. 1.Original synthetic image, 2. corrupted by artificial speckle noise with variance equal to 0.3 , and results of the smoothing achieved by the methods 3.median filter, 4. average filter, 5. Perona and Malik Anisotropic diffusion filter, 6. proposed selective average filter, 7. DPAD method, 8. 2D Bilateral filter, 9. Frost filter, 10.Wiener filter.

Concluding remarks are, proposed method save the evolution among the various structures of input image and making the region homogeneous with less variation in intensity

Sometime patches has been observed in ultrasound images which are (patches or entire image) affected by speckle noise (also known as multiplicative noise), hence the pixels inside these patches are taken into processing by estimating similarity (geometry) amongst the picture element, using by measuring the Kolmogorov-Smirnov distance between their distributions, proposed by Fabio Baselice [16][2017]. Suppose a multiplicative noise model is-

$$
\mathrm{z}(\mathrm{x}, \mathrm{r}, \mathrm{t})=\mathrm{y}(\mathrm{x}, \mathrm{r}, \mathrm{t}) \mathrm{n}(\mathrm{x}, \mathrm{r}, \mathrm{t}), \mathrm{x} \varepsilon \mathrm{X}, \mathrm{r} \varepsilon \mathrm{R}, \mathrm{t} \varepsilon \mathrm{T},\{\mathrm{X}, \mathrm{R}, \mathrm{T}\} \subset \mathrm{R}
$$

$\mathrm{y}($.$) represents noiseless signal, \mathrm{n}($.) represents speckle noise (usually Rayleigh distributed), (x,r) represents space indexes belongs to acquisition geometry defined in domains $X$ and $R, t$ 
represents acquisition time possess values in $\mathrm{T}$ domain. Reorganizing eq. (1) for a noiseless signal $y($.$) assuming time independent, can be written as-$

$$
\mathrm{z}(\mathrm{x}, \mathrm{r}, \mathrm{t})=\mathrm{y}(\mathrm{x}, \mathrm{r}, \mathrm{t}) \mathrm{n}(\mathrm{x}, \mathrm{r}, \mathrm{t})
$$

The Rayleigh distribution can be characterize for a captured signal $z(t)=y n(t)$ as below, by considering spatial position of pixel at $\left(\mathrm{x}_{0}, \mathrm{r}_{0}\right)$

$$
\mathrm{f}_{\mathrm{z}}(\mathrm{z} ; \mathrm{y}, \sigma)=\frac{z}{y \sigma 2} \exp \frac{-z 2}{2 y 2 z 2}
$$

Where $\sigma$ is the scale parameter of the Rayleigh probability density function.

Eq.(3) represents, the statistical behaviour of captured signal depends only on noise parameter $\sigma$ which is assumed constant for complete acquisition and noise free value y.

The cumulative Distribution Function (CDF) of z can be achieved by integrating eq. (3)

$$
\mathrm{F}_{\mathrm{z}}(\mathrm{z} ; \mathrm{y}, \sigma)=\mathrm{y}\left[1-\exp \left(\frac{-z 2}{2 y 2 z 2}\right)\right]
$$

A similarity measurement of two pixels $z(x 1, r 1, t)$ and $z(x 2, r 2, t)$ can be evaluated using Kolmogorov-Smirnov (KS) distance would be estimated using eq.(4), the distance $d$ of CDFs:

$$
d\left(\mathrm{x}_{1}, \mathrm{r}_{1}: \mathrm{x}_{2}, \mathrm{r}_{2}\right)=\max \left(\left|\hat{\mathrm{F}}_{\mathrm{z}(\mathrm{x} 1, \mathrm{r} 1)} \mathrm{F}_{\mathrm{z}}(\mathrm{z} ; \mathrm{y}, \sigma)-\hat{\mathrm{F}}_{\mathrm{z}(\mathrm{x} 2, \mathrm{r} 2)} \mathrm{F}_{\mathrm{z}}(\mathrm{z} ; \mathrm{y}, \sigma)\right|\right)
$$

A threshold of $(0,1)$ is applied for calculation of KS distance. For exploiting the CDF the picture element under process and neighboring pixels are taken into account. Experimental results of their method and other method for comparison are shown below-

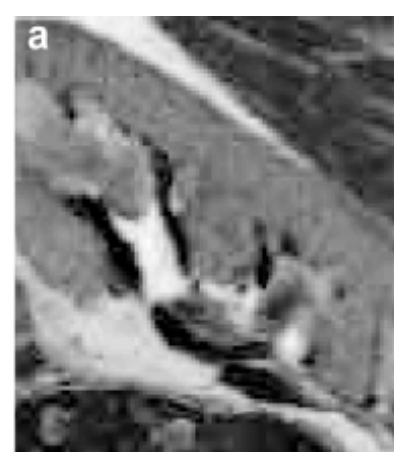

1

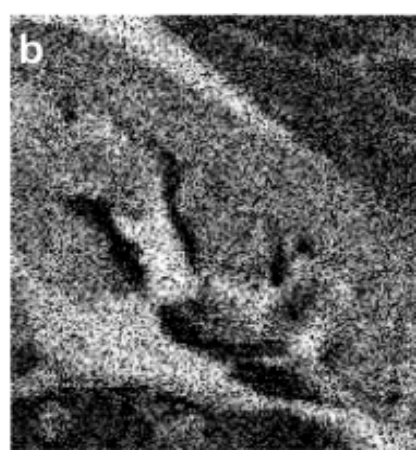

2

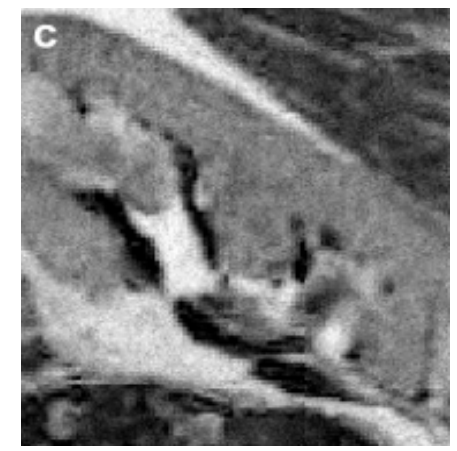

3 


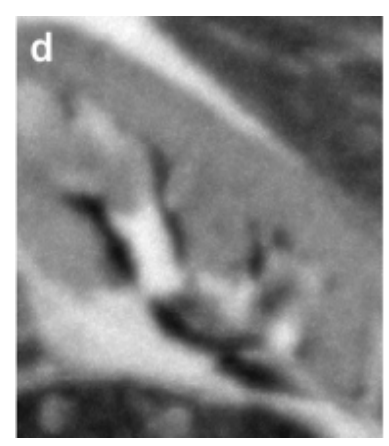

4

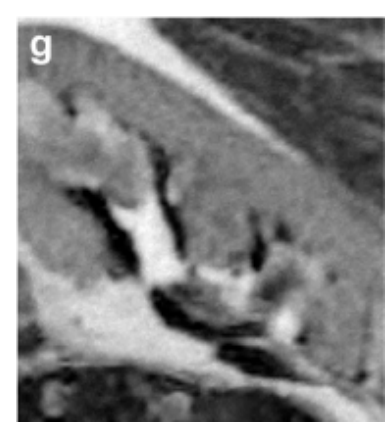

7

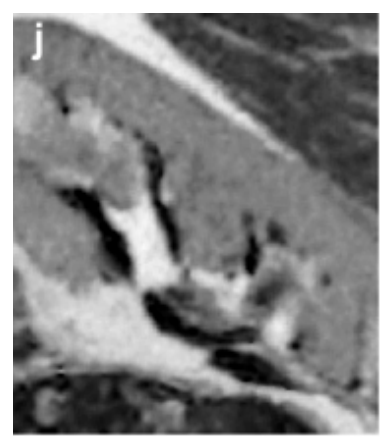

10

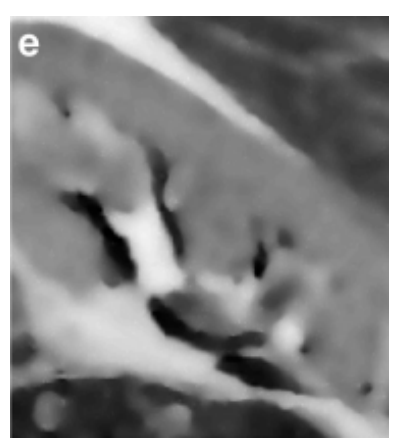

5

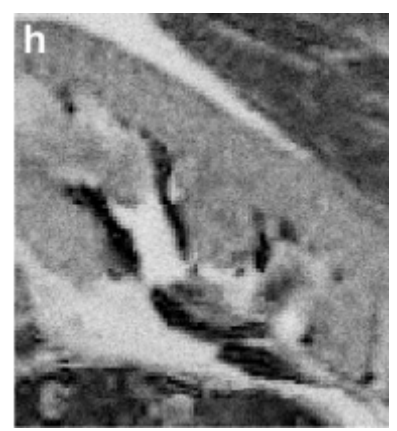

8

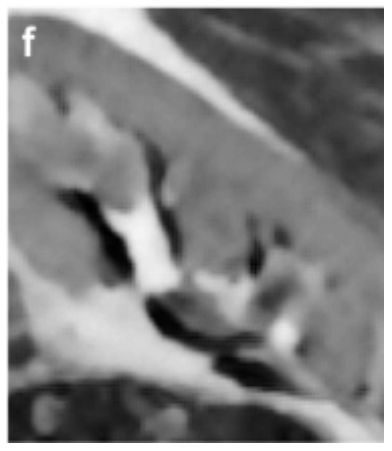

6

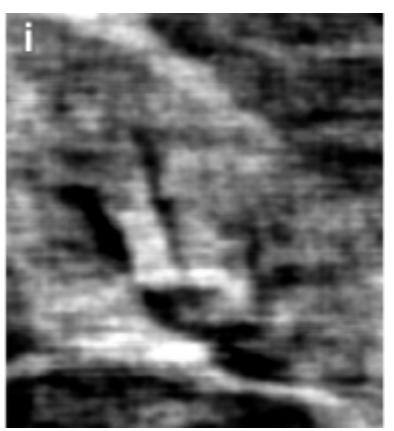

9

Fig. 2. Kidney simulated data set results, enlargement over a ROI: 1. reference image,2. noisy image, 3.mean image,4.anisotropic diffusion (AD),5.speckle-reducing anisotropic diffusion (SRAD),6. optimized Bayesian non-local mean (OBNLM), 7. 3-D maximum a posteriori (3D-MAP), 8.block matching 3-D (BM3D), 9.enhanced Wiener filter (EWF), 10.Proposed Kolmogorov-Smirnov non-local mean (KSNLM).

Concluding remarks are, merits of this method are, it can work pixel wise; it compare the behaviour of pixel with every other pixel in the image at location (x1,r1), instead of comparing it with patches of images. If a patch composed of few picture elements or one pixel, then filtering process will not be affected by ghost effect.

Obscure artifacts and data-correlated speckle are the two major epidemics of ultrasound images which degrade the quality of image and make processing difficult to extract information from it. A non-local total bounded variation (NLTBV) based model for removal of artifacts was proposed by P. Jidesh et. al.[17][2017]. This model regularizes the integrity of data using 
maximum a posteriori (MAP) and calculation of probability density function (PDF). A NLTV model was used to deal with piecewise linear approximation issue for restoring local gradient oscillation and removal of blurring artifacts. At the time of every iteration the regularization parameters were calculated like noise variance evaluated using Maximum Likelihood Estimate (MLE) of the input data. Speckles are data-correlated and can be modelled as

$$
\mathrm{u} 0=\mathrm{kun}
$$

here $\mathrm{n}$ indicates the multiplicative speckles following the Gamma law, $K$ is a linear bounded blurring operator. From the background of Aubert-Ajol model and non-local model of gamma distribution of speckle

$$
\min _{u}\left\{\int_{\Omega}\left|\nabla_{N L} u\right| d x d y+\lambda \int_{\Omega}\left(\log (K u)+\frac{u_{0}}{k_{u}}\right) d x d y\right\}
$$

This model was designed using MAP estimation method and substituting non-local total bounded variation norms with non-local TV norms, turns the equation into

$$
\min _{u}\left\{\int_{\Omega}\left(\left|\nabla_{N L} u\right|+\frac{\beta}{2}\|u\|^{2} L^{2}\right) d x d y+\lambda \int_{\Omega}\left(\log (K u)++_{K u}^{u 0}\right) d x d y\right\}
$$

Where $\beta$ represents a positive scalar constant that controls the magnitude of NLTV and $\mathrm{L}^{2}$ norms of image function $u$. Experimental results of proposed method and comparison with other methods are shown below-

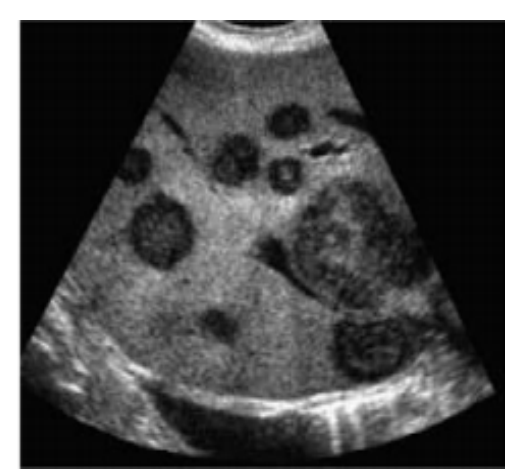

1

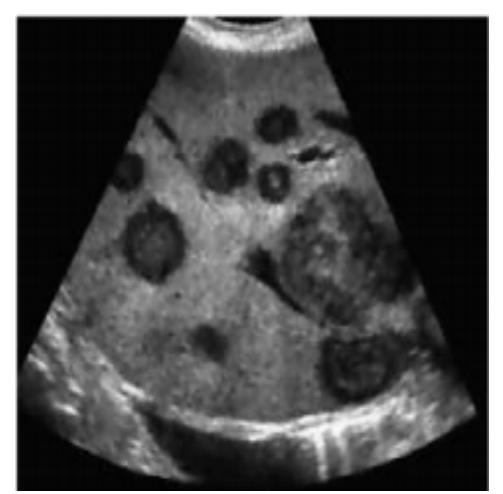

4

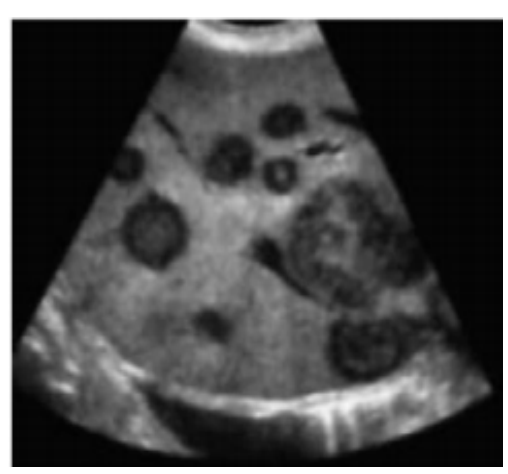

2

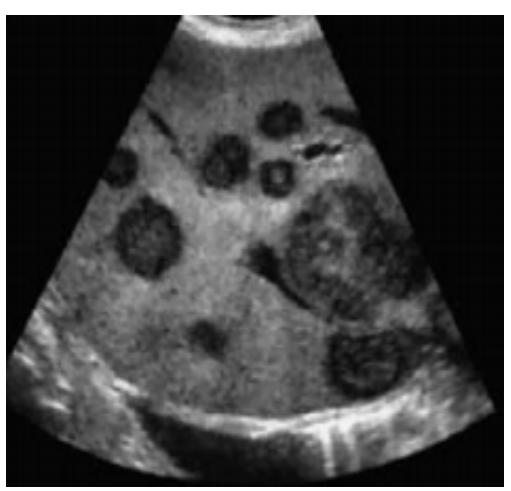

5

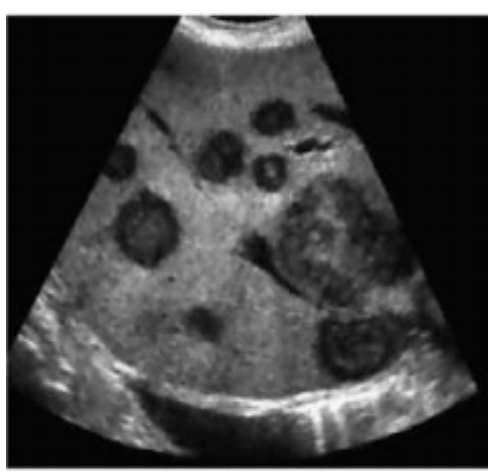

3

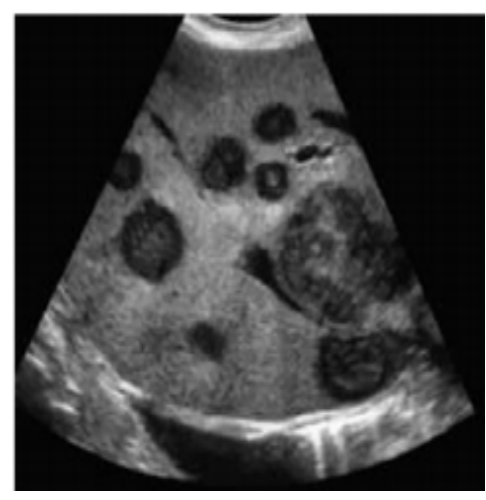

6 


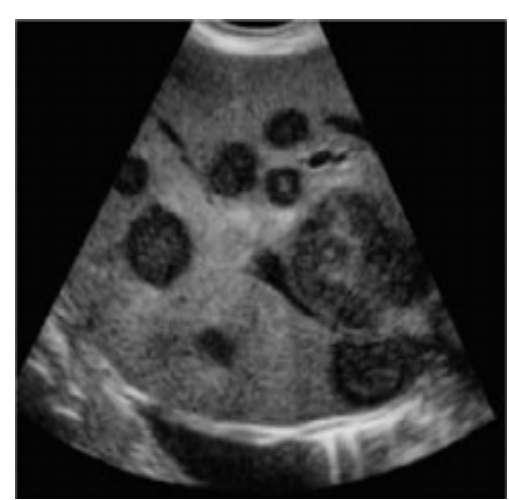

7

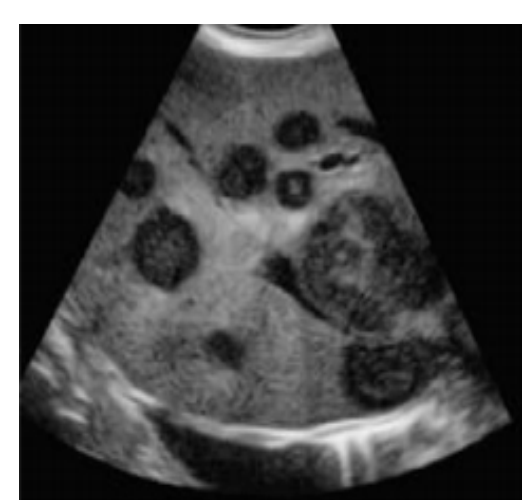

8

Fig. 3. 1. Original ultrasound image of human Liver (Speckled and Blurred image), 2. image restored by SRAD, 3. image restored by DPAD, 4. image restored by OSRAD, 5. image restored by AA model, 6.image restored by NLTV method, 7. image restored by NLTV using AL method, 8. image restored by the proposed method.

Concluding remarks are, the non-local TBV instead of NLTV offers excellent visual results to the restored output. Use of augmented Lagrangian approach enhances the rate of convergence of model and offers suitable to use in real time applications.

A despeckling method using guided filter and improved wavelet filter was proposed by Ju Zhang et. al. [18] [2015]. An emitted ultrasonic signal into human body when reflected back (this reflected signal usually known as envelope signal) accompanied with unwanted noisy signal (usually because of sensors), a model of envelope signal was presented as-

$$
f^{\operatorname{Pre}}(i, j)=g^{\operatorname{Pre}}(i, j) n^{\text {Pre }}(i, j)+w^{\text {Pre }}(i, j)
$$

here $\mathrm{f}^{\mathrm{Pre}}(\mathrm{i}, \mathrm{j})$ indicates envelope signal (or observed signal) of USG signal, $g^{\text {Pre }}(\mathrm{i}, \mathrm{j})$ indicates original signal, $n^{\text {Pre }}(i, j)$ indicates multiplicative noisy signal, $w^{\text {Pre }}(i, j)$ si an additive noise signal, and superscript pre represents preliminary signal. Considering the value of $w^{\mathrm{Pre}}(\mathrm{i}, \mathrm{j})$ is small compared to $n^{\text {Pre }}(\mathrm{i}, \mathrm{j})$ noisy signal, $\left(\left|\mathrm{w}^{\text {Pre }}(\mathrm{i}, \mathrm{j})\right|^{2}<<\left|\mathrm{n}^{\text {Pre }}(\mathrm{i}, \mathrm{j})\right|^{2}\right)$, the additive $\mathrm{w}^{\text {Pre }}(\mathrm{i}, \mathrm{j})$ can be skipped and eq. (1) can be written again as-

$$
f^{\operatorname{Pre}}(i, j)=g^{\text {Pre }}(i, j) n^{\text {Pre }}(i, j) \in Z^{2}
$$

Difference in resolution of imaging system, the distribution of speckle will also differ. The multiplicative noise $n^{\text {Pre }}(i, j)$ follows Rayleigh distribution. If the noise calculation parameter is signal to noise ratio, multiplicative noise can be modelled using Rician distribution. The filtration of additive noise can be done through wavelet denoising procedure. Dynamic range of monitor is a biggest hurdle in compression of envelope signal, hence log transform is applied 
before wavelet transform to achieve effective compression ratio of an envelope signal. Eq. (2) becomes additive noise model where $g^{\text {Pre }}(i, j) n^{\text {Pre }}(i, j)$

$$
\log \left(f^{\text {Pre }}(i, j)\right)=\log \left(g^{\text {Pre }}(i, j)\right)+\log \left(n^{\text {Pre }}(i, j)\right)
$$

$\log \left(f^{\text {Pre }}(i, j)\right)$ represents USG image data, $\log \left(n^{\text {Pre }}(i, j)\right)$ represents white (Gaussian) noise.

2 dimensional DWT (Discrete Wavelet Transform) of a noise affected image can be processed using an orthogonal wavelet transform for a $2 \mathrm{D}$ image. Eq.(3) can be re-written in wavelet domain as below-

$$
W_{l, k}^{j}(f)=W_{l, k}^{j}(g) W_{l, k}^{j}(n)
$$

Where $\mathrm{j}=1,2, \ldots, \mathrm{J}, 1, \mathrm{k}, \in \mathrm{Z}^{2}$. All three above stated parameters of $\mathrm{f}, \mathrm{g}, \mathrm{n}$ in eq.(4) represents coefficients of noise affected image, noiseless image and data-correlated noise in wavelet domain. Superscript and subscript J and 1,k represents layers and coordinates of wavelet domain. For ease of presentation author had rewrites the eq.(4) as

$$
F_{l, k}^{J}=G_{l, k}^{J}+N_{l, k}^{J}
$$

The use of Rayleigh model for cleaning speckle noise from USG images as-

$$
\mathrm{P}_{\mathrm{N}}(\mathrm{n})=\frac{n}{\sigma_{n}^{2}} \exp \left(-\frac{n 2}{2 \sigma_{n}^{2}}\right)
$$

$\sigma_{n}$ represents standard deviation of speckle noise in wavelet domain.

The hybrid algorithm comprising guided filter with wavelet shrinkage algorithm uses a threshold function to enhance the noise removal ratio and storing maximum details of image, for devising an innovative concept of compression Bayesian maximum a posteriori was used, and for removal of noise in low frequency sub band a guided filter was designed and used.

Experimental results of proposed method and comparison with existing method are shown below-

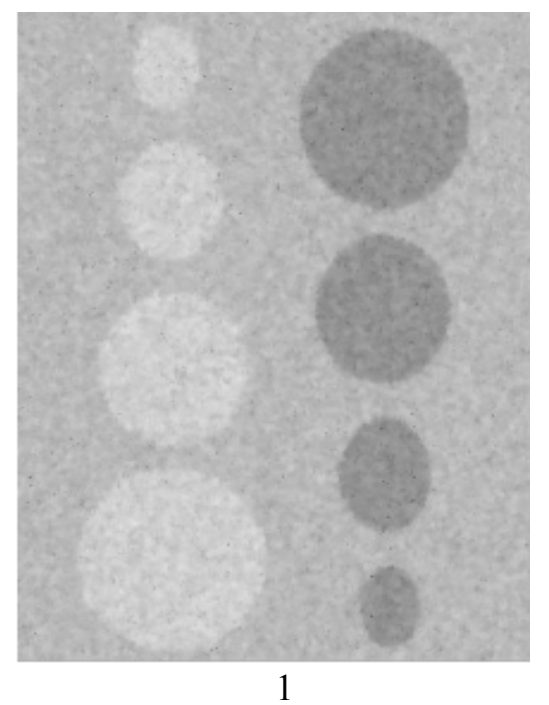

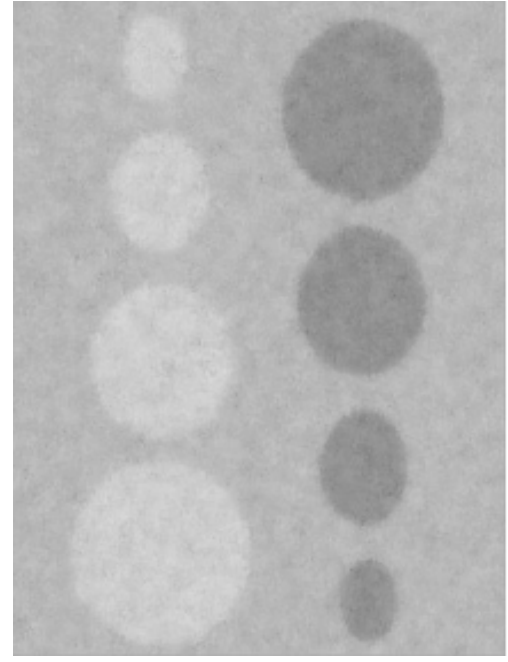

2

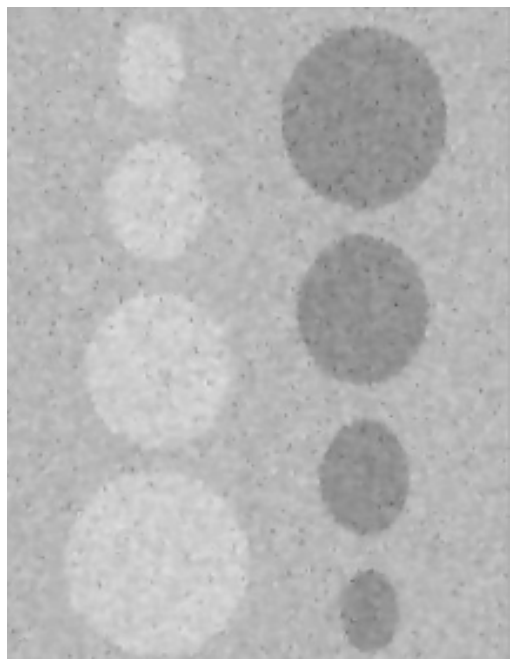

3 


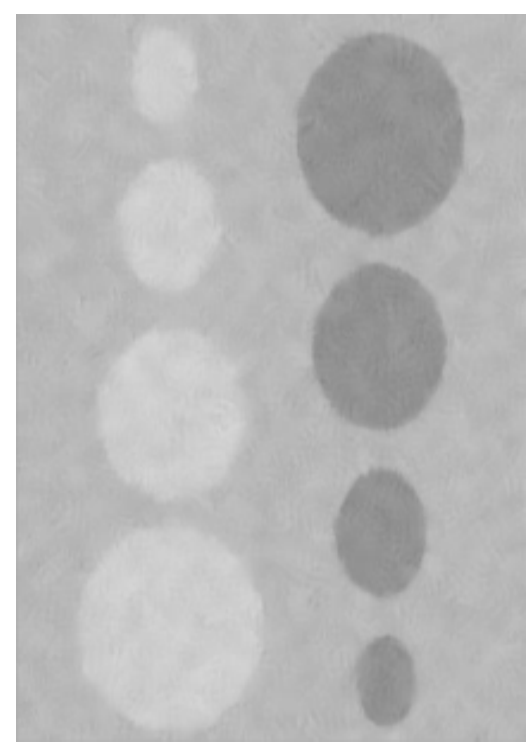

4

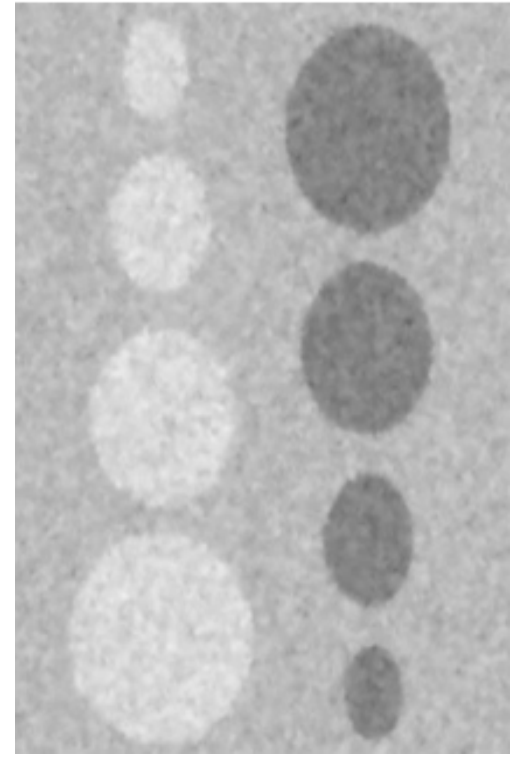

7

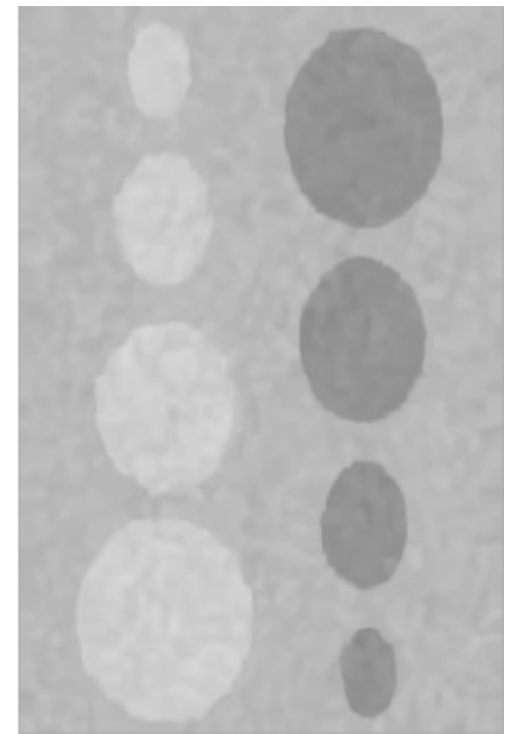

5

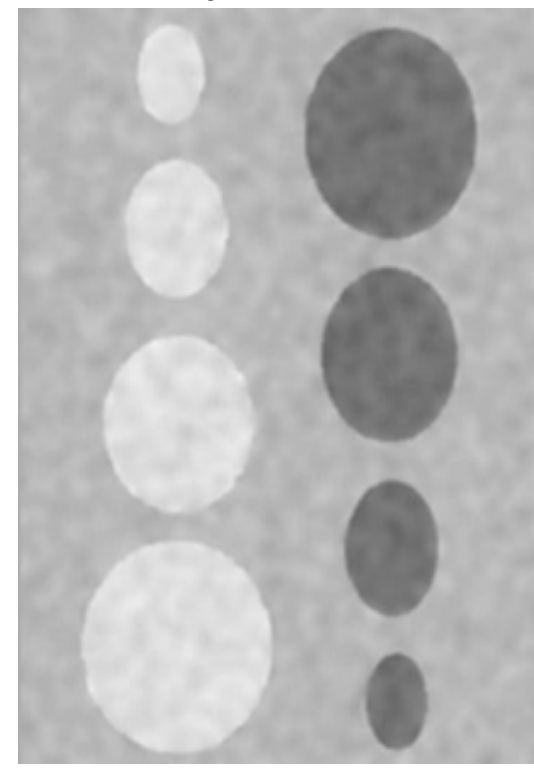

8

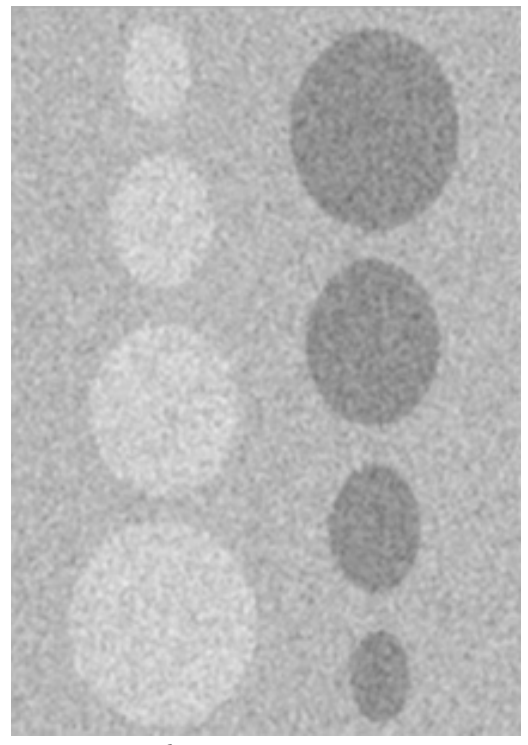

6

Fig. 4. The de-noised images of simulated ultrasound image experiment, 1. Bilateral filter, 2.Guided filter, 3.DTD filter, 4.OBNLM method, 5.SAR-BM3D method, 6.Wavelet soft, 7. WaveletBilateral,8.Proposed method.

Concluding remark is, the suggested method has good edge preservation capability with excellent speckle removing feature.

For despeckling from USG images two methods that are subset of weighted nuclear norm minimization (WNNM) based upon low-rank approximation technique were proposed by Sameera V. et. al. [19][2017]. First technique uses homomorphic filtering using low rank minimization technique and second method used to increase the efficiency of despeckling process at pre-processing stage. Assume a speckle noise as below-

$$
\mathrm{N}=\mathrm{M} \eta
$$


Here $\eta$ indicates multiplicative speckle noise, $\mathrm{N}$ indicates noise free image and $\mathrm{M}$ indicates image affected by speckle noise. For the application of low rank approximation method for despeckling process, first multiplicative noise must be converted into additive format, for this log transform was used,

$$
\begin{aligned}
\mathrm{Y} & =\log (\mathrm{N}) \\
& =\log (\mathrm{M})+\log (\eta) \\
& =\mathrm{X}+\eta
\end{aligned}
$$

Here $X$ indicates original image processed by $\log$ transform \& $\eta$ indicates additive noise (Gaussian noise).

USG

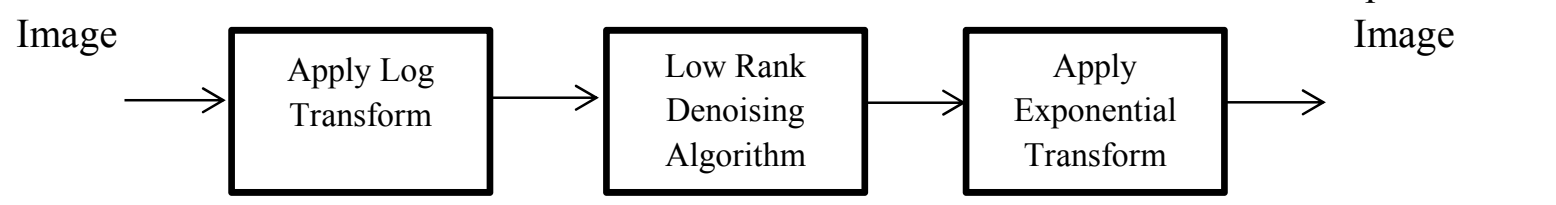

Fig.5: Block diagram of DLRA process

When the eq.(2) is about to processed by WNNM method, hence it is processed by two subsets of it, first one is known as Grouping, of identical section having p-dimension into a data structure of $\mathrm{p}+1$ dimension. For reconstruction of an image, non-local self-similar patches (NSS) helps.

Second is Matching, used to regulate a patch come by computing nearest patch between reference position and patch at different spatial location. Patch with shortest distance from reference are counted as similar and clustered together. Suppose size of patch Yi be mxm retrieved from $Y$, i indicate co-ordinate of top left corner of block. Suppose two patches $Y_{i} \& Y_{j}$. Patch $\mathrm{Yj}$ is identical to $\mathrm{Yi}$ if the distance amongst them is less than a set threshold; the distance can be defined as-

$$
\mathrm{d}\left(\mathrm{Y}_{\mathrm{i}}, \mathrm{Y}_{\mathrm{j}}\right)=\frac{\|(\mathrm{Yi}, \mathrm{Yj})\|_{2}^{2}}{m^{2}}
$$

Let $\mathrm{Y}_{\mathrm{i}}$ be the all similar non-local patch grouped together, hence

$$
\mathrm{Y}_{\mathrm{j}}=\mathrm{X}_{\mathrm{j}}+\mathrm{n}_{\mathrm{j}}
$$

Here $n_{j}$ and $Y_{j}$ indicates patches of noises and noise free image and $X_{j}$ represents a low rank matrix.

Weighted nuclear norm minimization is a low rank approximation technique used to calculate the image free from noise effect as result from above stated process. The WNM model was created as below- 


$$
\hat{X}_{\mathrm{j}}=\arg \min X_{j} \frac{1}{\sigma_{n}^{2}}\left\|\mathrm{Y}_{\mathrm{i}}-\mathrm{Y}_{\mathrm{j}}\right\|_{F}^{2}+\left\|\mathrm{X}_{\mathrm{j}}\right\|_{\mathrm{w}, *}
$$

Here $\sigma_{n}^{2}$ indicates variance of noise and is added to normalize the fidelity term, $\left\|Y_{i}-Y_{j}\right\|_{F}^{2}$ indicates $\mathrm{F}$ - norm data fidelity term. If noise has low variance it increase the the fidelity term during denoising procedure and vice-versa.

Experimental results of proposed method and comparison with existing method are shown below-

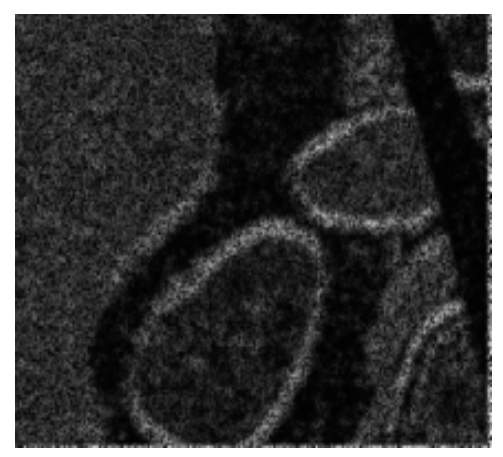

1

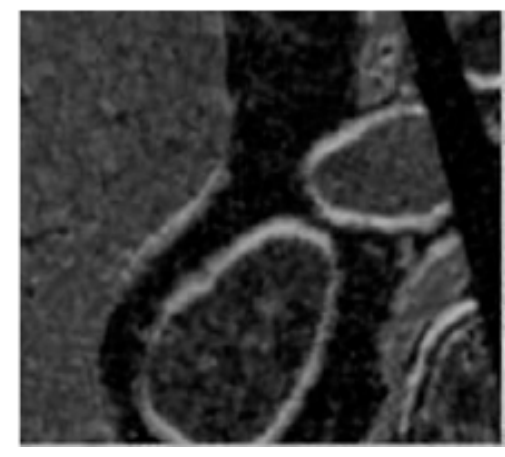

2

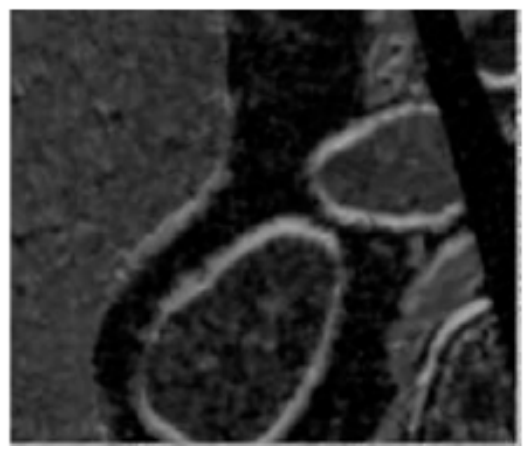

3

Fig. 6. De-noised results of simulated ultrasound experiment (simulated image), 1. noisy image, 2. DLRA ,3. Modified DLRA.

Concluding remarks are, the input for DLRA method was the time gap between successive video frames of ultrasound imaging system. The modified DLRA method was incorporated the algorithm for processing the statistical properties of ultrasound image, because of its speckle removing capability and excellent capability of restoring of edges.

Without setting a threshold value despeckling of ultrasound image can be done using a bio inspired multi-gene genetic based programming (MGGP) algorithm was proposed and implemented by Syed Gibran Javed et.al. [20] [2017]. This algorithm was based upon parallel gene structure with the use of statistical features of surrounding pixels. Their methodology works on two stages feature extraction stage and despeckling of USG images using MGGP algorithm. Noise corrupted images D were applied as an input for feature extraction stage. A $3 \times 3 \& 5 \times 5$ sized blocks were created from noise affected image, features consist of information of interest were extracted using these blocks and create a feature vector $\mathbf{u}$, which hold several statistical parameters of an image. $\mathbf{u}$ is also an input to next stage for denoising purpose. Here the parallel structure of multi-gene has been used for despeckling process.

For despeckling process 10-dimensional feature vectors were devised in feature extraction stage. In the view of pre-stated fitness criterion, MGGP looks for optimum solution. At the time of evolution cycle estimation function looks for discriminant features from vector $\mathbf{u}$. The region of interest was identified by feature vector $\mathbf{u}=\left[\mathrm{u}_{1}, \mathrm{u}_{2}, \mathrm{u}_{3}, \mathrm{u}_{4}, \mathrm{u}_{5}, \mathrm{u}_{6}, \mathrm{u}_{7}, \mathrm{u}_{8}, \mathrm{u}_{9}, \mathrm{u}_{10}\right]$ and the target value $f$ 
extracts from noiseless image F. Training data set was used for generation of estimation function $\hat{f}(\mathrm{u})$ as shown below

$$
S_{u}^{T r}=<u^{(m)}, f^{(m)}>_{m=1}^{M T r}
$$

Here $u^{(m)}$ indicates $m^{\text {th }}$ feature vector belongs to target $f^{(m)} \in \mathrm{F}$.

For determination of performance testing dataset was used as shown below-

$$
S_{u}^{T s}=<u^{(m)}, f^{(m)}>_{m=1}^{M T s}
$$

Here $M T s$ indicates testing and $M T r$ indicates training data sets respectively.

Several function set were defined during the evolution cycle for estimator, which consist of several trigonometric and arithmetic operators for several variables and constants. This process was named as defining primitive operator for MGGP. The selection of fitness function was as per nature of problem. For despeckling error should be minimum between image affected by noise and image without image, root mean square error ((RMSE) as a fitness function was used, as shown below-

$$
\operatorname{RMSE}=\sqrt{\frac{\Sigma(f(x, y)-\widehat{f}(\mathrm{x}, \mathrm{y}))}{M}}
$$

Here $\hat{f}(\mathrm{x}, \mathrm{y})$ Indicates estimated values of picture elements, and $f(x, y)$ indicates target values of picture element. Precise design of fitness function guarantee the success of evolution phase. During the despeckling process minimization of error against a tolerance level suggest the end of process. This criteria of end of process not met then healthiest gene from the population was identified for operator (like reproduction, cross over, mutation) selection for new evolution cycle. When termination criterion was achieved, simulation will be stopped.

The multi-gene $I_{G P}^{e s}(u) \rightarrow g^{e s}$ having best fitness isdefined as speckle denoising estimator. This estimation was selected to transform ensemble gene $g=\left[g_{1}, g_{2}, \ldots . . g m\right]$ to target $f(x, y)$ by reducing error between $\mathrm{f}(\mathrm{x}, \mathrm{y}) \in \mathrm{F}$.

Experimental results of proposed method and comparison with existing method are shown below- 


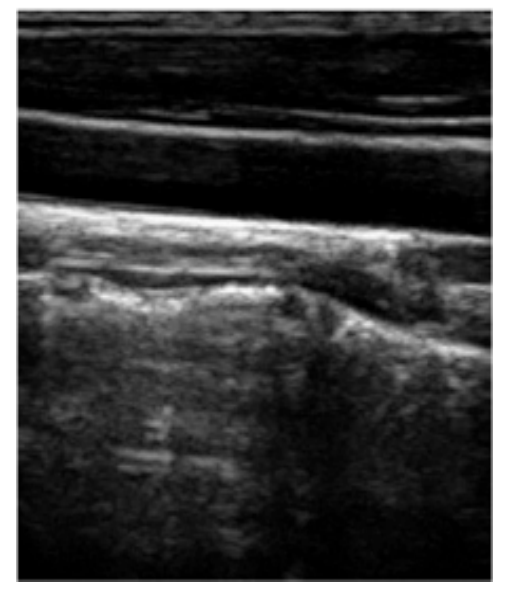

1

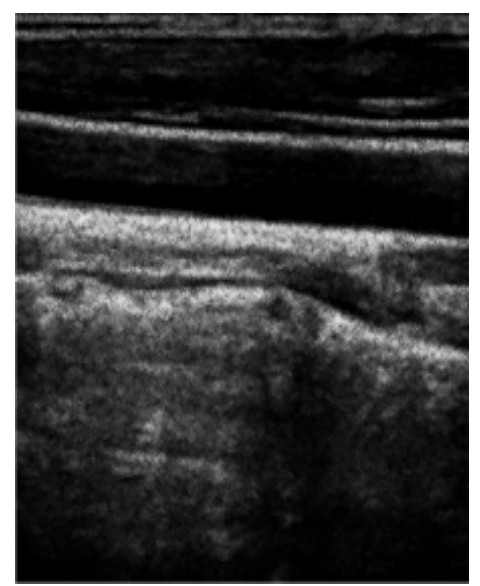

4

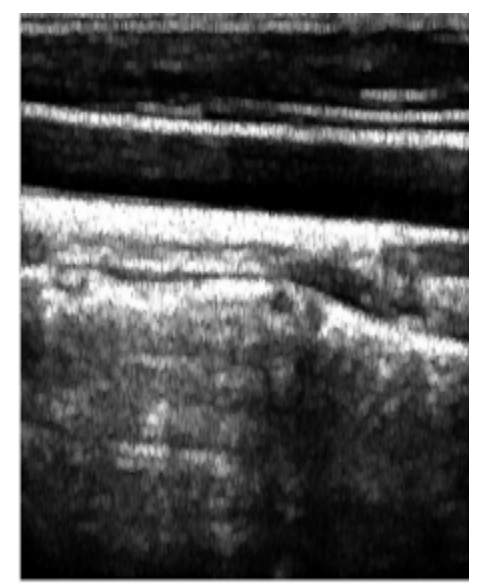

7

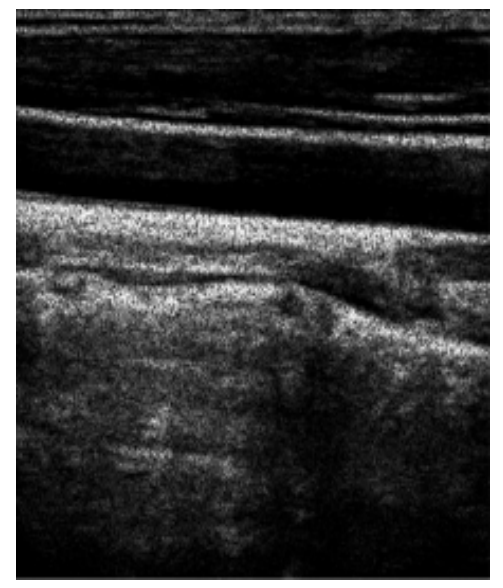

2

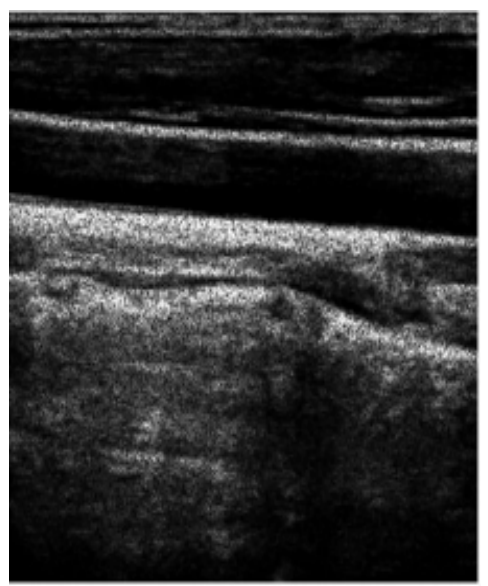

5

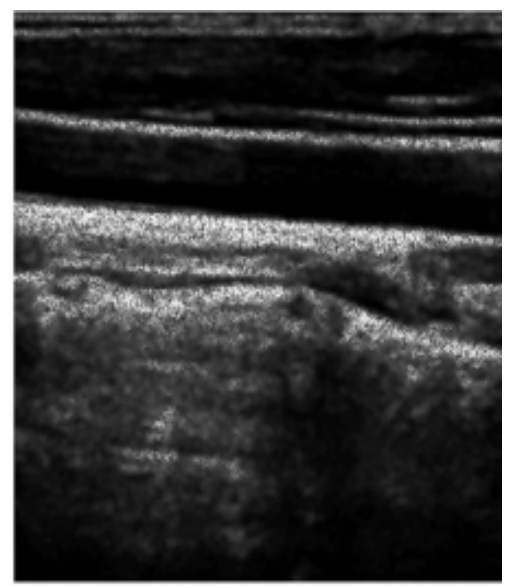

8

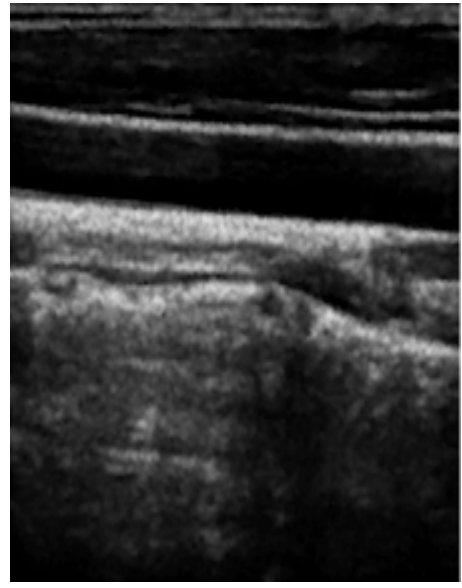

3

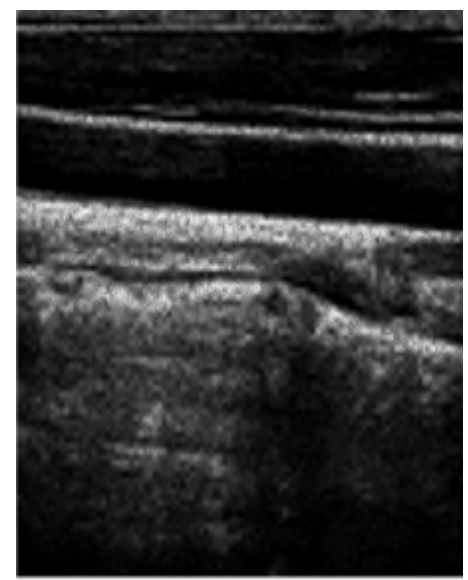

6

Fig. 6. Visual comparison of images corrupted with 0.23 variance, 1.Original image, 2. noisy image, 3. Proposed method, 4. LSMV, 5. SRAD, 6. Wavelet, 7. GF, 8.Wiener

Concluding remarks are, merits of this method states, it does not require log transform of corrupted image also does not require any threshold. 
For despeckling of ultrasound images a hybrid algorithm consist of multiscale variance stabilization technique uses Fisz transform along with hyperbolic wavelet transform (HWT) was implemented by Younes Farouj et. al. [21] [2017]. The HWT preserves the original details of image. Their method uses Nadaraya-Watson estimator technique which does not require any prior knowledge of noise model being implied. The multiplicative noise was first converted into additive noise model using-

$$
\mathrm{v}=\mathrm{u}+\mathrm{e}
$$

$\mathrm{v}$ indicates output image, $\mathrm{u}$ indicates unknown image and e is random noise components. The demerit of model shown in eq.(1) is, it does not consider that the intensity of image is proportional to occurrence of noise. Considering this fact it is modified model states that noise component does not remain constant, hence

$$
\mathrm{v}=\mathrm{u}+\mathrm{u}^{\gamma} \mathrm{e}
$$

e represents zero-mean Gaussian white noise, and $\gamma>0$. Model (2) is suitable for motion estimation and useful for speckle modeling. $\gamma$ can be used for latching the statistical details of image inside the scanner after post-processing step. Model (1) in wavelet domain can be re-written as -

$$
\mathrm{d}_{\mathrm{j}, \underline{\mathrm{k}}}(\mathrm{v})=\mathrm{d}_{\mathrm{j}, \underline{\mathrm{k}}}(\mathrm{u})+\mathrm{d}_{\mathrm{j}, \underline{\mathrm{k}}}(\mathrm{e})
$$

The thresholding estimator

$$
\hat{u}_{\sigma}=\sum_{\mathrm{j}, \mathrm{k \varepsilon I} \sigma} \mathrm{dj}, \mathrm{k}(\mathrm{v}) \psi_{\mathrm{i}, \underline{\mathrm{k}}}
$$

While restoring an image, they modeled the unknown parameters of images as different dimensions to different parameters of image. To deal with such kind of situation hyperbolic wavelet transform was implemented.

Experimental results of proposed method and comparison with existing method are shown below-

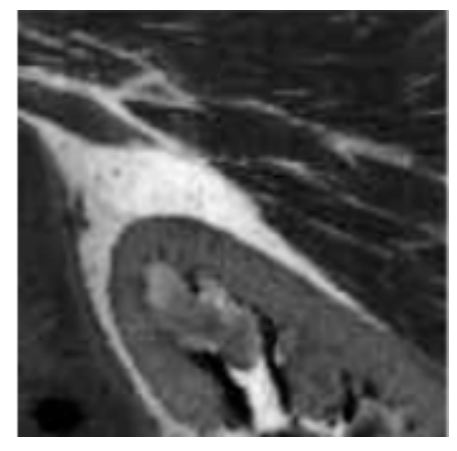

1

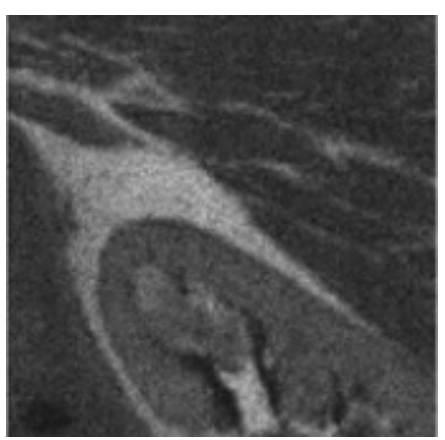

2

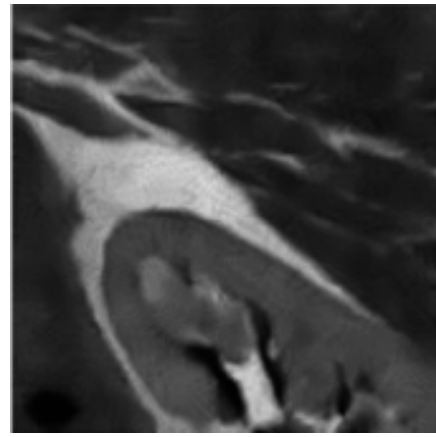

3 


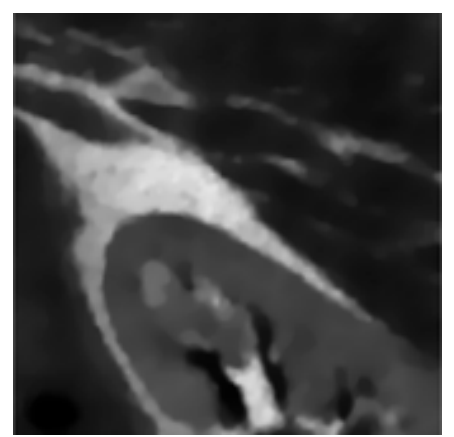

4

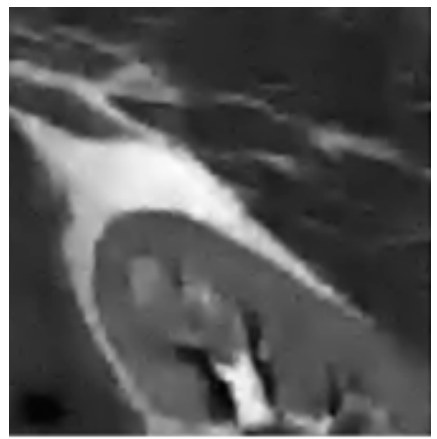

5

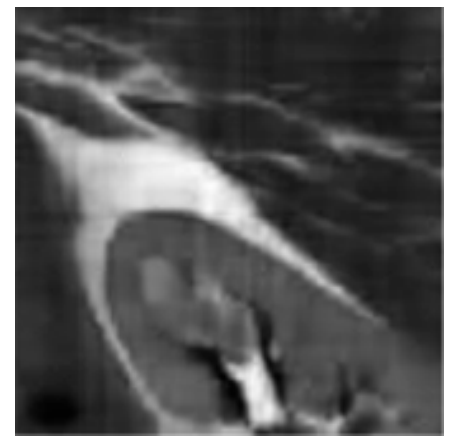

6

Fig.7. Results of different methods applied to the Kidney image, 1.original image, 2.corrupted image, image processed by 3. OBNLM method, 4.variational method, 5.IWF, 6. HWF

Concluding remarks are, the wavelet-Fisz (WF) method was applied using the nondecimated wavelet transform (NDWT). And extension to data driven method known as HWF and data driven hyperbolic WF method (dHWF) was employed over an image affected by pepper noise. The loss of data was found proportional to amount of noise present in the image. Window size $\mathrm{M}=8$ applied over $\mathrm{n}$ image of 256x256 size, HWT was applied for 40.72 seconds and dHWf was applied for 54.51 second on a system with core i7 processor.

For despeckling, two step procedure involving use of fractional integration filter and fuzzy weighted mean was proposed and implemented by Ayesha Saadia et. al.[22]. The diversity in pixel intensity is one of the important factor used to recognize pixels from particular regions such as edge, homogeneous and detailed, of an image. When a particular pixel is corrupted by multiplicative, simultaneously neighboring pixels are also get affected equally. A fuzzy logic with a window size of $3 \times 3$ is used to process a pixel under consideration. Window was placed over central pixel, its value and value of pixels covered by window, their weighted mean was taken and it will be a new value of central pixel. If the difference of value between pixels is large, pixels are part of different regions; else they are part of same region. A fuzzy set of two intensity values MORE and LESS was used. They considered and image with difference in intensity value as 0 and $255, \mathrm{X}(\mathrm{J})$ is a variation in intensity value of image $\mathrm{A}(\mathrm{I})$ and $\mathrm{Y}(\mathrm{T})$ defines level in variation of intensity value, $Y=\{$ LESS, MORE $\}$. Pixels with difference in intensity les than 40 will be allocated fuzzy function LESS, and pixels with high intensity difference (or based upon the difference in increment in pixel intensity, the member ship value goes decline), in these scenario the fuzzy function MORE is allocated. A is having value between 1 and 0.1 represent full membership \& 0 indicates no membership. A maximum function was used in overlapped section, and the value of membership was calculated using- 


$$
\begin{array}{ll}
\mu_{\text {LESS }}=1 & \mathrm{~J} \leq 40 \\
\mu_{\mathrm{LESS}}=\frac{170-X}{170-40} & 40<\mathrm{X}<170 \\
\mu_{\mathrm{MORE}}=\frac{X-40}{170-40} & 40<\mathrm{X}<170 \\
\mu_{\mathrm{MORE}}=1 & 170 \leq \mathrm{X} \leq 255
\end{array}
$$

For determining the weight of the pixel $(i, j)$

$$
\mathrm{w}(\mathrm{i}, \mathrm{j})=\begin{array}{ll}
\mu_{\text {LESS }} & \mu_{\text {LESS }} \geq \mu_{\text {MORE }} \\
1-\mu_{\text {MORE }} & \mu_{\text {LESS }} \leq \mu_{\text {MORE }} \\
0.1 & \mu_{\text {MORE }}=1
\end{array}
$$

For substituting new weighted value of pixel under process-

$$
\mathrm{R}(\mathrm{a}, \mathrm{b})=\frac{\sum_{i=-Q}^{Q} \cdot \sum_{j=-Q}^{Q} \mathrm{w}(\mathrm{i}, \mathrm{j}) \mathrm{xI}(\mathrm{a}+\mathrm{i}, \mathrm{b}+\mathrm{j})}{\sum_{i=-Q}^{Q} \cdot \sum_{j=-Q}^{Q} \mathrm{w}(\mathrm{i}, \mathrm{j})}
$$

$R(a, b)$ represents processed image of step-1, w(i,j) represents weight coefficient, $I(a+i, b+j)$ represents pixels in window of size $(2 \mathrm{Q}+1) \mathrm{x}(2 \mathrm{Q}+1), \mathrm{i}, \mathrm{j} \varepsilon[-\mathrm{Q}, \mathrm{Q}], \mathrm{Q}=1$ was taken.

In second step for despeckling process, image $\mathrm{R}$ was processed by fractional filter. GrunwaldLetnikov (G-L) fractional differential was used. A fractional integration mask of size $3 \times 3$ was applied in directions $0^{\circ}, 45^{\circ}, 90^{\circ}, 135^{\circ}, 180^{\circ}, 225^{\circ}, 270^{\circ}$ and $315^{\circ}$, resultant was added to obtained a mask of size $5 \times 5$.

Concluding remarks are, merit of their proposed method is, it restore the edge with maximum information during and after the despeckling process.

Interpolated Cartesian images result of uncompressed ultrasound data corrupted by speckle noise and represented by Gamma distribution. For removal of speckle from these type of images a model based on non-local means (NLM) was proposed and implemented by P.V. Sudeep et. al. [23]. A new class of non-local means filter known as unbiased non-local means filter (ULNM) subtracted from LNM filter response. B-mode scan was model uses three parameter Gamma distribution as shown in below eq.-

$$
\begin{aligned}
& \Gamma(\mathbf{x} \mid \gamma, \rho, \beta)=\left\{\begin{array}{cc}
\frac{(x-\gamma)^{\rho-1}}{\Gamma(\rho) \beta^{\rho}} \exp \left(-\left(\frac{x-\gamma}{\beta}\right)\right) & \forall \gamma<\mathbf{x}<\infty, \quad \rho>0, \beta>0 \\
0 &
\end{array}\right. \\
& \text { otherwise }
\end{aligned}
$$

$\Gamma$ (.) represents Gamma function, $\gamma$ is parameter indicates location, $\rho$ represents parameter for shape and $\beta$ represents parameter for scale.

For despeckling of every pixel 
$\hat{u} \mathrm{NLM} \mathrm{u}((\mathrm{r}))=\sum_{\forall s \varepsilon \Omega} w(r, s) u(s)$

$\sum_{\forall s \varepsilon \Omega} w(r, s)=1,0 \leq w(r, s) \leq 1$, here s indicates each pixel inside the search window $\Omega$.

The weight $w(r, s)$ can be stated as-

$w(r, s)=\frac{1}{Z(r)} e^{-\frac{d(r, s)}{h^{2}}}$

$\mathrm{H}$ regulate the smoothing and computed as a $\mathrm{SD}$ (standard deviation of noise). $\mathrm{Z}(\mathrm{r})$ is a normalizing constant and can be expressed as-

$\left.\left.\mathrm{Z}(\mathrm{r})=\sum_{\forall s \varepsilon \Omega} e^{(-(} d(r, s)\right) / h^{2}\right)$

d represents Gaussian weighted Euclidean distance of all pixels covered by search window.

$\mathrm{d}(\mathrm{r}, \mathrm{s})=\mathrm{G}_{\sigma}\left\|\mathrm{Br}_{\mathrm{r}}-\mathrm{B}_{\mathrm{s}}\right\|_{t 2}^{2}$

$\mathrm{G}_{\sigma}$ Gaussian weighting function (normalized) with $\sigma \mathrm{SD}$ and mean $=0 . \mathrm{B}_{\mathrm{r}}$ and $\mathrm{B}_{\mathrm{s}}$ represents gray level value matrix of $U$ at pixels of window centered at $r$ th and sth pixel. When $r=s$ leads to over weighting effect because of self similarity is very high. To deal with such type of situation-

$\mathrm{w}(\mathrm{r}, \mathrm{r})=\max (\mathrm{w}(\mathrm{r}, \mathrm{s}) \quad \forall \mathrm{s} \neq \mathrm{r})$

Eq.(6) represents NLM filter specifically designed for despeckling.

For calculation of unbiased value of every pixel

$\operatorname{NLM}$ speckle $(U)=\max \left(\operatorname{NLM}(U)-\hat{\rho}_{\text {ML }} \hat{\beta}_{\text {ML }, 0 \mathrm{~s})}\right.$

Experimental results of proposed method and comparison with existing method are shown below-

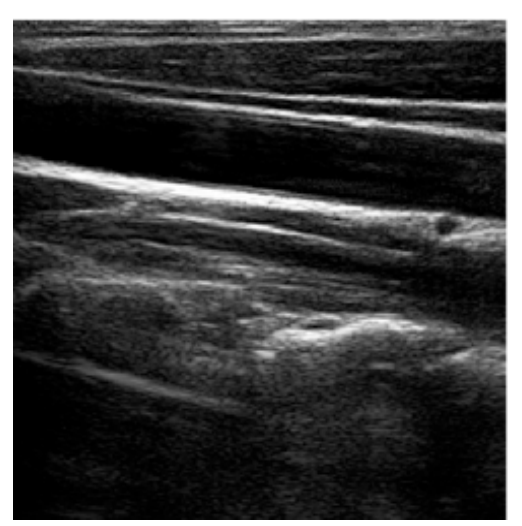

1

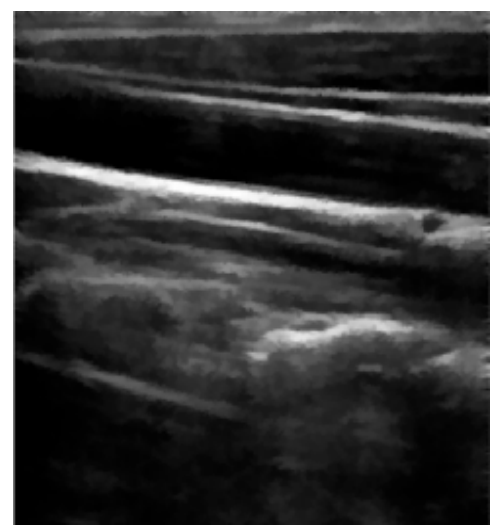

2

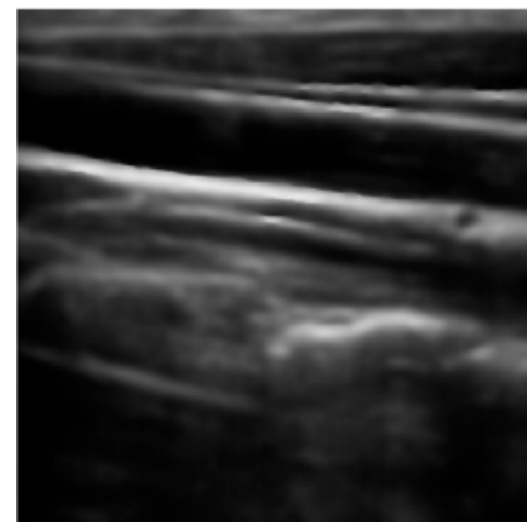

3 


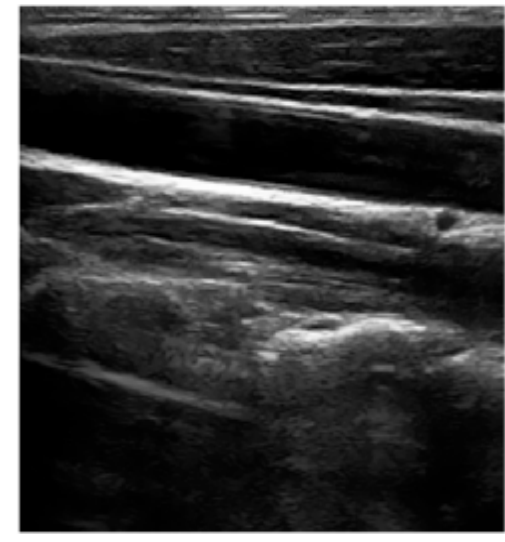

4

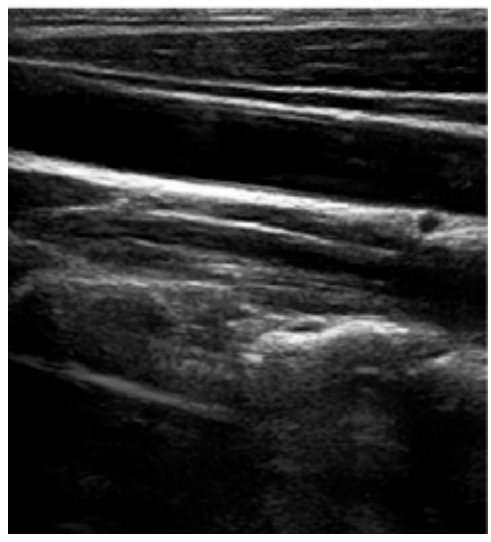

5

Fig. 8. Results obtained with different filters applied to the real B-mode carotid US image, 1. Real image, 2.denoised result with SBF Filter, 3.denoised result with SRAD Filter, 4.denoised result with OBNLM Filter, 5. denoised result with Proposed NLM Filter.

Concluding remarks are, proposed method have automatically estimated parameter for shape, scale, and other parameter such as window size and similarity window size.

The demerit of this filter is large computational time si required for despeckling.

For despeckling, a hybrid algorithm, which segment the image into regions like texture, edge, smooth with the implementation of coefficient of variation, was proposed and implemented by Ayesha Saadia et.al. [24][2017]. A fuzzy logic data set is used to sketch a boundary between these regions. Same filter won't provide desired output for all the regions of an image, hence their algorithm select different order of fractional filter for different regions of image for despeckling of pixel. To sketch a line between different regions, fuzzy function was used. An image $\mathrm{H}$ is affected by speckle noise can be expressed as-

$$
\mathrm{H}(\mathrm{x}, \mathrm{y})=\mathrm{G}(\mathrm{x}, \mathrm{y}) \cdot \mathrm{N}(\mathrm{x}, \mathrm{y})
$$

$\mathrm{G}(\mathrm{x}, \mathrm{y})$ is an original image, $\mathrm{N}(\mathrm{x}, \mathrm{y})$ indicates speckle noise, $\mathrm{H}(\mathrm{x}, \mathrm{y})$ indicates image free from speckle noise having dimension $\mathrm{MxN}, \mathrm{x}=1 \ldots \mathrm{M}$ and $\mathrm{y}=1 \ldots \mathrm{N}$

For classification of an image, uses of coefficient of variation (CV) defined as

$$
\mathrm{CV}=\frac{\mathrm{SD}}{\text { mean }}
$$

SD indicates standard deviation, and mean indicates arithmetic mean of pixels blankets by a $3 \times 3$ windows. Pixel having small value of $\mathrm{CV}$ belongs to smooth region, high value of $\mathrm{CV}$ is for edges and medium value is for texture regions. The fuzzy membership value of every pixel can be calculated by: 


$$
\mathrm{H}(\mathrm{x}, \mathrm{y}) \varepsilon\left\{\begin{array}{lc}
\mu_{\text {smooth }}=\frac{c-C V}{c-a} & a \leq C V \leq m 1 \\
\mu_{\text {texture }}=\max \left(\min \left(\frac{C V-m 1}{c-m 1}, \frac{m 2-C V}{m 2-c}\right), 0\right) & m 1<C V \leq m 2 \\
\mu_{\text {edge }}=\frac{C V-c}{b-c} & \text { otherwirse }
\end{array}\right.
$$

Threshold values a, b, c, m1, m2 can be estimated by-

$$
\begin{gathered}
\mathrm{a}=\min \left(\mathrm{CV}_{\mathrm{i}}\right) \\
\mathrm{b}=\max \left(\mathrm{CV}_{\mathrm{i}}\right) \\
\mathrm{c}=\operatorname{median}\left(\text { unique }\left(\mathrm{CV}_{\mathrm{i})}\right)\right. \\
\mathrm{m} 1=\operatorname{average}\left(\mathrm{CV}_{[\mathrm{a}, \mathrm{c}]}\right) \\
\mathrm{m} 2=\text { average }\left(\mathrm{CV}_{[\mathrm{c}, \mathrm{b}]}\right)
\end{gathered}
$$

Smooth regions have high frequency components and employing a fractional filter will add complexity to denoising process, hence they have used average filter and for pixels belongs to texture and edges fractional integral filter was used. Pixels corrupted by noise in smooth regions were processed by a window of size $3 \times 3$, and average of value of all the pixels will gives new value for central pixel using below equation:

$$
\mathrm{G}^{\prime}(\mathrm{x}, \mathrm{y})=\frac{1}{9} \sum_{i=-1}^{1} \cdot \sum_{j=-1}^{1} H(x+i, y+j)
$$

$\mathrm{G}^{\prime}$ represents the probable noise free image.

Experimental results of proposed method and comparison with existing method are shown below-

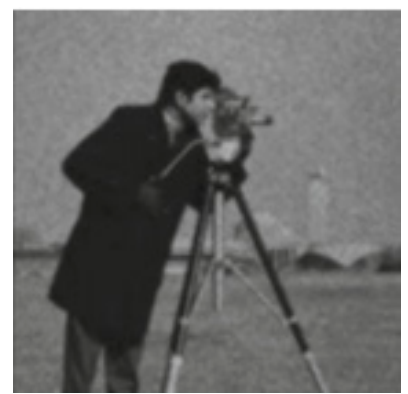

1

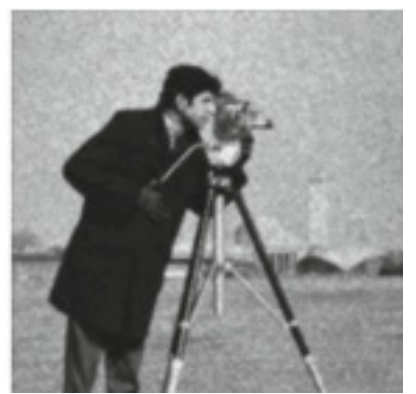

2

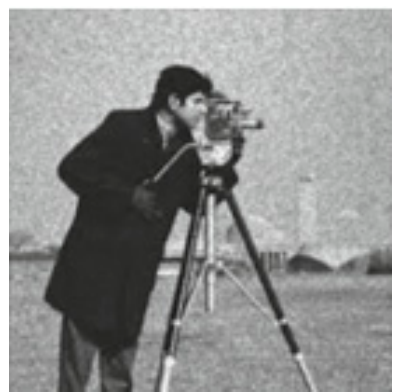

3 


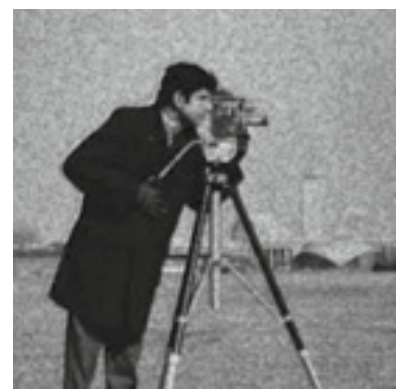

4

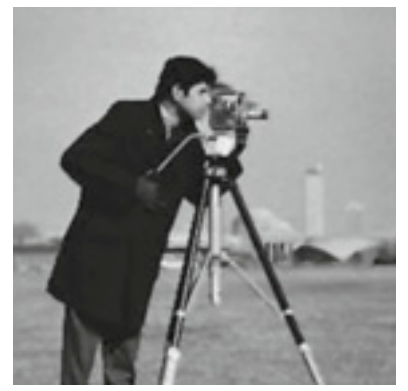

7

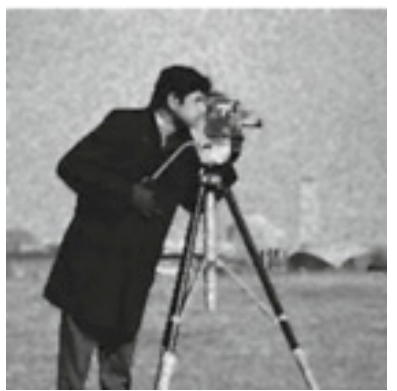

5

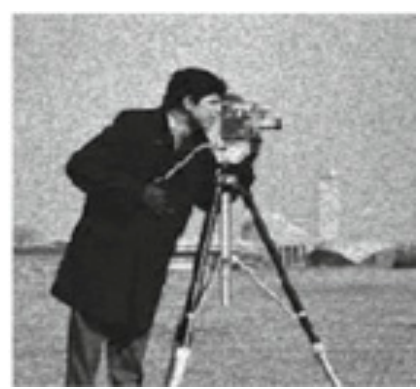

8

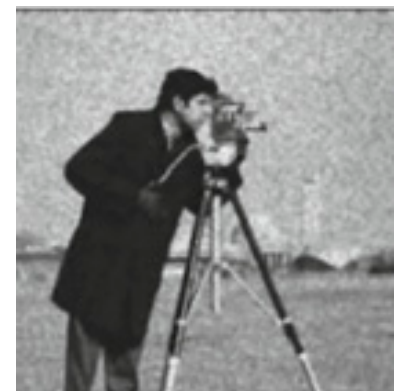

6

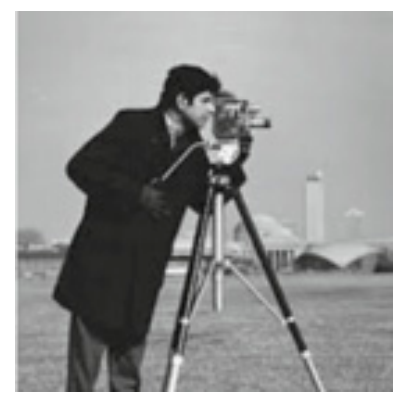

9

Fig. 9. Denoising results Cameraman image with noise variance 0.02 , 1. Riemann-Liou method, 2. fractional order method, 3. improved fractional-order method,4. adaptive fractional calculus, 5. local statistics, 6.Adaptive method, 7. Proposed method, 8.noisy image, 9. original Cameraman image

Concluding remarks are, merits of proposed method includes, along with promising despeckling result restoration of edge details and based upon the nature of region filter is applied (either fractional or adaptive filter).

Most of the denoising algorithm treat image information as noise and process it along with it, which results into loss of image data. Principle of fuzzy logic was used by Jayachandran Jai Jaganath Babu et. al. [25] [2016], for classification of corrupted pixels into edge, homogeneous and details sections. Parameters required for classification was derived from corrupted pixels. An adaptive filter adjusts itself based upon the type of region under process. This adaptiveness was employed at two levels, at first level, for classification of images; coefficients of variation (CV) were calculated from the corrupted image. At second level, a weighted average filter was used for classification between noise and edges. The fuzzy filtering process was performed as: Detection process, in which Coefficient of Variation as a parameter used to group pixels into several subdomains. Filtering process: involves use of three filters for noise removal and image enhancement. Computed CV values were mapped to fuzzy domain through Gaussian membership function:

$$
\mu_{m}^{i}(u)=e^{\left(-(u-c i)^{2} / 2 \sigma_{i}^{2}\right)}
$$


$\mathrm{i}=1,2,3, \mathrm{c}_{\mathrm{i}}$ represents means and $\sigma_{\mathrm{i}}$ represents variance of three regions. Threshold of three class of regions edges, details and homogeneous can be expressed as a, b, c as given in below eq.:

$$
\mu_{m}^{i}(u)=\left\{\begin{array}{rr}
\text { edge } & u>b \\
\text { detail } & u \geq a \text { and } u \leq c \\
\text { homogeneous } & u<b
\end{array}\right.
$$

Based upon the value of presence of noise across a pixel, threshold a, b, c changes.

Five IF-THEN rules of fuzzy domain was used for classification as shown below-

\begin{tabular}{|c|c|c|c|c|}
\hline Rule & Fuzzy set & Large & Small & Region \\
\hline \multirow[t]{3}{*}{1} & $\mu_{m}^{1}(u)$ & $\checkmark$ & & \multirow[t]{3}{*}{$\mathrm{H}$} \\
\hline & $\mu_{m}^{2}(u)$ & & $\checkmark$ & \\
\hline & $\mu_{m}^{3}(u)$ & & $\checkmark$ & \\
\hline \multirow[t]{3}{*}{2} & $\mu_{m}^{1}(u)$ & & $\checkmark$ & \multirow[t]{3}{*}{$\mathrm{D}$} \\
\hline & $\mu_{m}^{2}(u)$ & $\checkmark$ & & \\
\hline & $\mu_{m}^{3}(u)$ & & $\checkmark$ & \\
\hline \multirow[t]{3}{*}{3} & $\mu_{m}^{1}(u)$ & & $\checkmark$ & \multirow[t]{3}{*}{$\mathrm{E}$} \\
\hline & $\mu_{m}^{2}(u)$ & & $\checkmark$ & \\
\hline & $\mu_{m}^{3}(u)$ & $\checkmark$ & & \\
\hline \multirow[t]{3}{*}{4} & $\mu_{m}^{1}(u)$ & $\checkmark$ & & \multirow[t]{3}{*}{$\mathrm{D}$} \\
\hline & $\mu_{m}^{2}(u)$ & $\checkmark$ & & \\
\hline & $\mu_{m}^{3}(u)$ & & $\checkmark$ & \\
\hline \multirow[t]{3}{*}{5} & $\mu_{m}^{1}(u)$ & & $\checkmark$ & \multirow[t]{3}{*}{$\mathrm{E}$} \\
\hline & $\mu_{m}^{2}(u)$ & $\checkmark$ & & \\
\hline & $\mu_{m}^{3}(u)$ & $\checkmark$ & & \\
\hline
\end{tabular}

$\mathrm{H}=$ homogeneous, $\mathrm{D}=$ detail $\mathrm{E}=$ edge

The denoising phase uses three different filters to process three distinctive regions, for homogeneous region a smoothening filter of window size $(2 \mathrm{~K}+1) \times(2 \mathrm{~K}+1)$ was used, this filter takes average of all the pixel values, under the window and assigned it as a new value $F(x, y)$, to pixel over which the mask is placed. The new value of pixel post averaging process will be expressed as:

$\mathrm{F}(\mathrm{x}, \mathrm{y})=\frac{1}{(2 K+1)^{2}} \sum_{r=-k}^{k} \cdot \sum_{s=-k}^{k} R^{\prime}(x+r, y+s)$

$\mathrm{k}=1,2, \ldots$. Is an integer. 
For detail region median filter of window size $(2 \mathrm{~K}+1) \times(2 \mathrm{~K}+1)$ was used, for restoring details of edges.

$\mathrm{F}(\mathrm{x}, \mathrm{y})=\operatorname{median}\left(R^{\prime}(x+r, y+s)\right) \quad$ where $-\mathrm{k} \leq(\mathrm{r}, \mathrm{s}) \leq \mathrm{k}$

$\mathrm{k}=1,2, \ldots$. is an integer.

Edge region has the prime requirement that, during the despeckling process, no loss of boundary data of an image is bearable; hence to achieve this objective, adaptive weighted average filter was designed and implemented, in consideration of points like, pixels which are grouped together and possess constant intensity are assigned higher weights. Pixel corrupted in nature are separate and discrete in nature and various intensity values, hence less valued weight is assigned. It is expressed as:

$\mathrm{F}(\mathrm{x}, \mathrm{y})=\frac{\sum_{r=-k}^{k} \sum_{s=-k}^{k} w(r, s) x R^{\prime}(x+r, y+s)}{\sum_{r=-k}^{k} \sum_{s=-k}^{k} w(r, s)}$

$\mathrm{w}(\mathrm{x}, \mathrm{y})=\mathrm{m}(\mathrm{x}, \mathrm{y}) \mathrm{x} \mathrm{s}(\mathrm{x}, \mathrm{y})$

$\mathrm{m}(\mathrm{x}, \mathrm{y})$ represents identical magnitude and $\mathrm{s}(\mathrm{x}, \mathrm{y})$ identical spatial property of pixel.

$\mathrm{m}(\mathrm{x}, \mathrm{y})=\exp \left(-\left(\frac{R^{\prime}(x, y)-R^{\prime}(x+r, y+s)}{\delta}\right)^{2}\right)$

$\mathrm{s}(\mathrm{x}, \mathrm{y})=\exp \left(-\left(\frac{r 2+s 2}{(2 k+1) 2}\right)\right)$

where $\mathrm{R}^{\prime}(\mathrm{x}, \mathrm{y})$ indicates pixel under process, and $\mathrm{R}^{\prime}(\mathrm{x}+\mathrm{r}, \mathrm{y}+\mathrm{s})$ indicates neighboring pixels, covered by a window of size $(2 \mathrm{k}+1) \mathrm{x}(2 \mathrm{k}+1)$.

$\mathrm{r}, \mathrm{s} \varepsilon[-\mathrm{k}$ to $\mathrm{k}], \delta=\mathrm{C} \sigma_{\mathrm{n}} ; \delta$ separate out edge pixels and corrupted pixels, $\mathrm{C}$ is tuning factor, $\sigma$ is variance.

Experimental results of proposed method and comparison with existing method are shown below- 


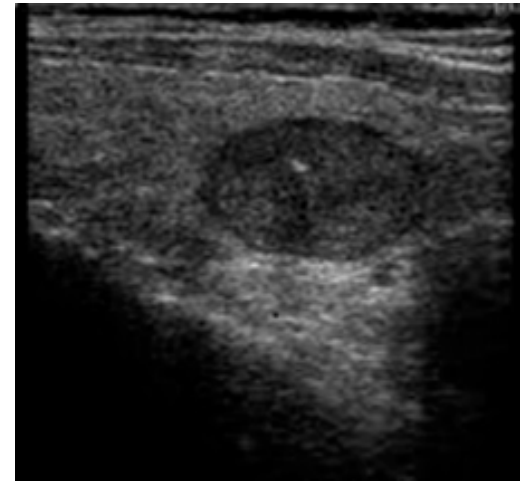

1

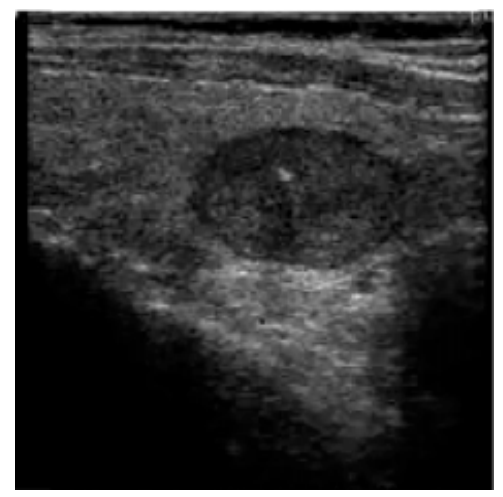

4

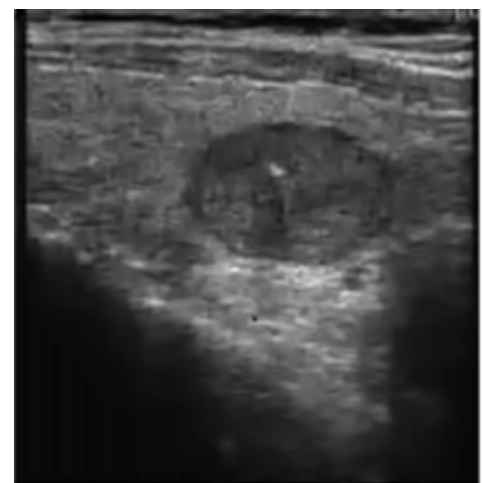

2

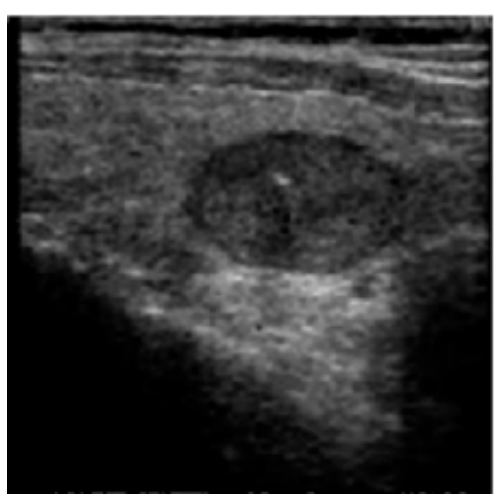

5

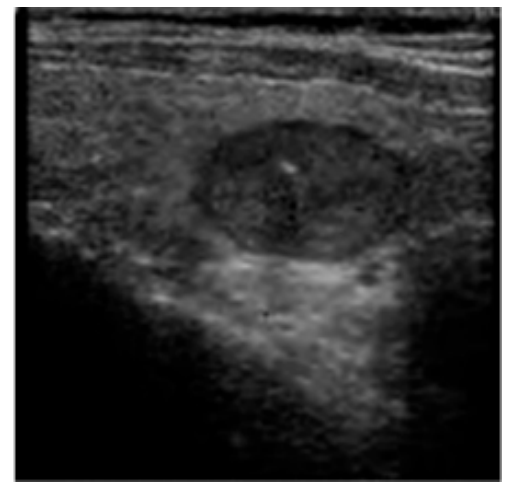

3

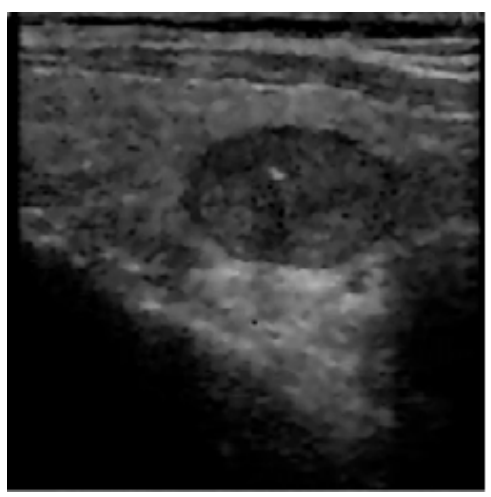

6

Fig. 10. Different denoising methods applied on ultrasound thyroid image,1.ultrasound thyroid image, 2 . GenLik, 3.SNIG I, 4.adaptive bilateral filter, 5. ATMAV, 6. proposed adaptive fuzzy logic filter based on CV.

Concluding remarks are, merits of proposed method includes, algorithm adaptively select an algorithm for filtering as per the nature of region being processed, and algorithm define a threshold adaptively based upon type of image being processed.

When medical images are infected by speckle noise, information contents and contrast level both are greatly affected in such a way that, it leads to false result which ultimately affect the resulting capability of medical practitioner. A hybrid algorithm for noise removal at local and nonlocal data was proposed and implemented by karamjeet Singh et. al. [26] [2017]. It works in three stages, at first stage a guided filter was used to cutback for despeckling. A guided filter has relationship between guidance $I$ and output $q$. Filter output $q$ provides a linear transformation of $I$ at a window $w k$ centered at pixel $k$.

$q_{i}=a_{k} I i+b_{k} \quad i \varepsilon w_{k}$

where

$a_{k}, b_{k}$ are linear and constant coefficient in $w_{k}$.

Experimental results of proposed method and comparison with existing method are shown below- 


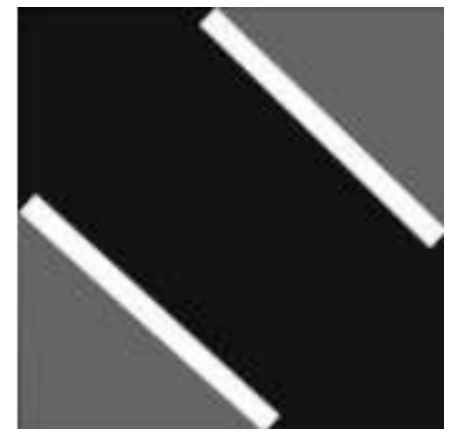

1

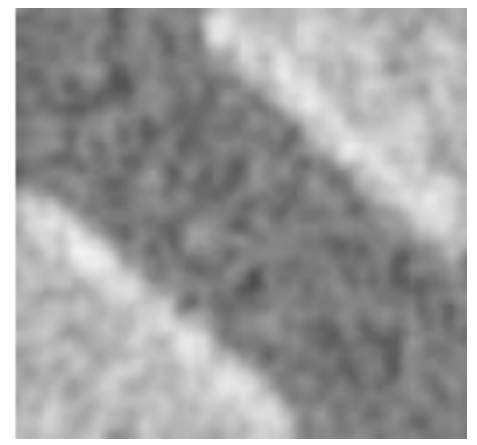

4

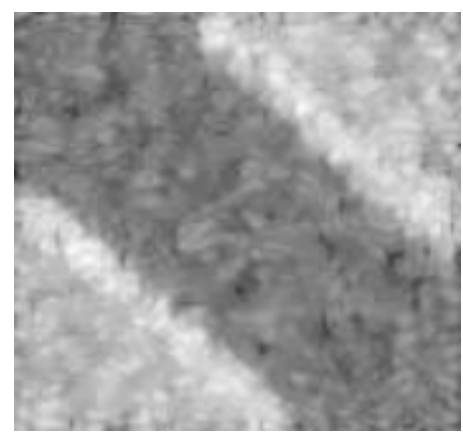

7

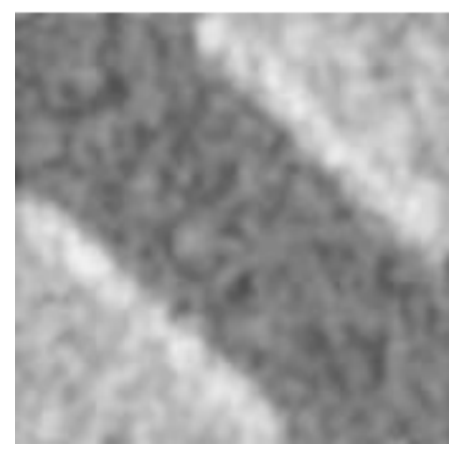

10

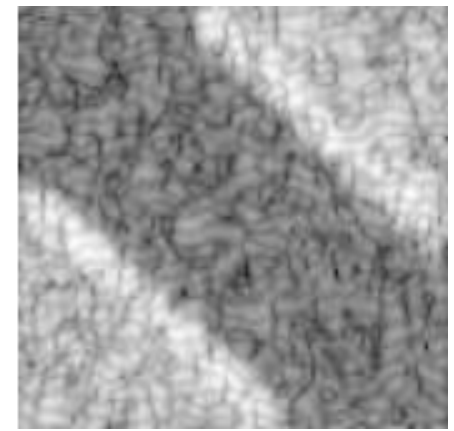

2

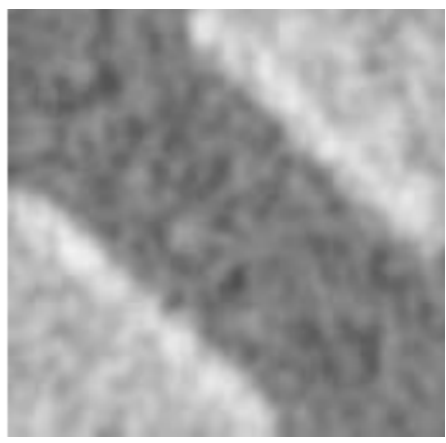

5

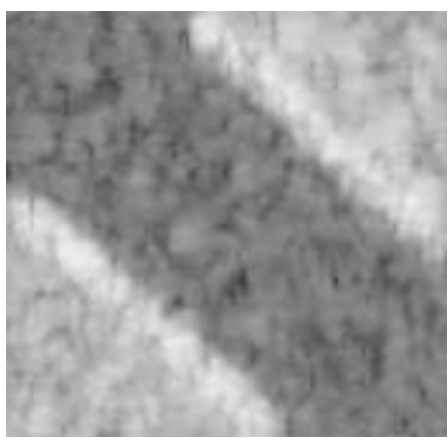

8

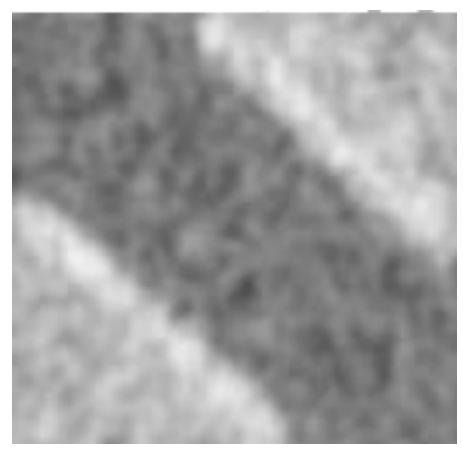

11

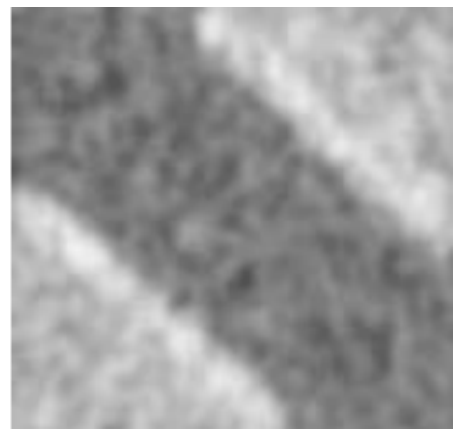

3

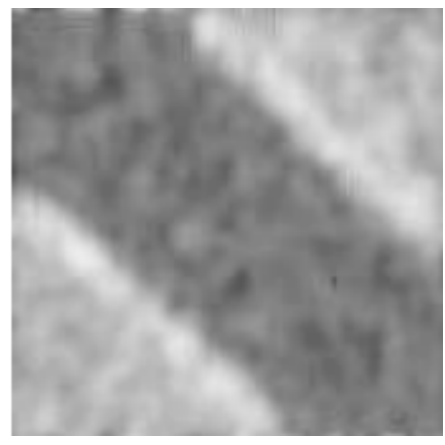

6

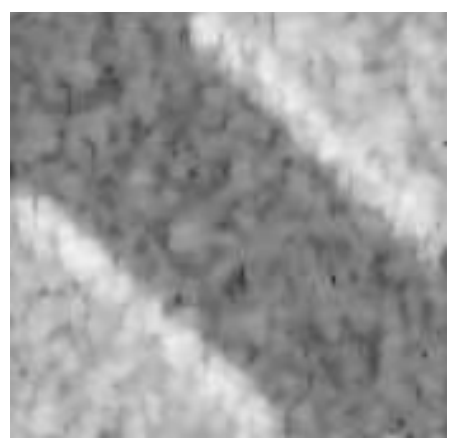

9

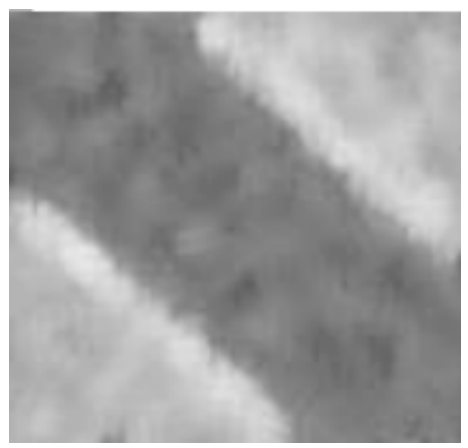

12 


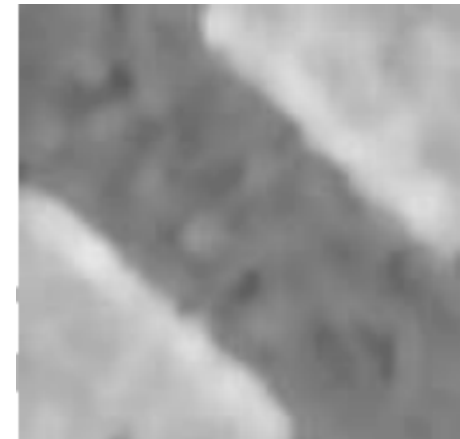

13

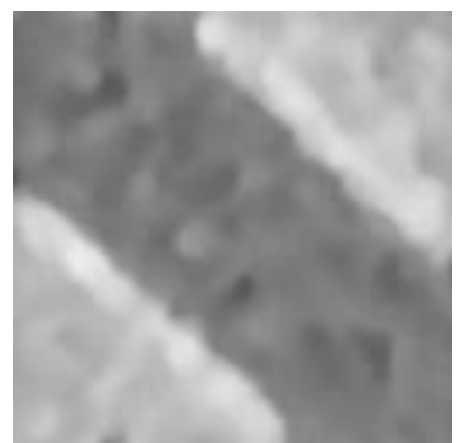

14

Fig. 11. Results of experiments:1. original image,2.Image corrupted with speckle noise with variance ; despeckled results of 3.Lee filter 4. Frost filter 5. Kaun filter 6. SRAD filter 7. SBF 8. GF 9. CBF 10. SRBF 11. GSRBF 12. CNLM filter 13. OBNLM filter 14. Proposed filter.

Concluding remarks are, an improved speckle reducing bilateral filter (SRBF) was developed to further reduce the speckle noise from the medical images. Adaptively removal of speckle noise capability was the prime reason for developing the bilateral filter, the second objective was restoration of anatomical details of organs. Hence to enhance the quality of image. At third stage a rotation invariant bilateral non local means (RIBNLM) filter was used because of its capability to detect sharp edges and sharp corner, to uplift the performance of denoising process. 


\section{Conclusion:}

From the above discussion, we can conclude that, some of the filters remove speckle noise successfully but unfortunately unable to restore the information at the edges and boundary of the objects. A two-step procedure used by weighted mean filter and fractional integration filter in fuzzy logic domain for achieving both above stated objectives. Most of the parts of USG images are corrupted by speckle effect, which deteriorates the quality of imaging and leads to difficulty in analysis and decision making. This method of filtration based upon concept of fuzzy logic restores the edge with maximum information during and after the despeckling process. In our opinion preserving edges of an ultrasound images can save lots of precious details.

\section{Compliance with Ethical Standards}

\section{Conflict of interest: The authors declare that they have no competing interests.}

Ethical approval: For this type of study formal consent is not required. 


\section{References:}

[1] Atam Dhawan, H K Huang, Dae-shik kim, Principles and Advanced Methods in Medical Imaging and Image Analysis, Medical Imaging and Image Formation, World Scientific Publishing Co. Pte. Ltd. Chapter 2, PP 9-28, 2008.

[2] Elisa Konofagou, Principles and Advanced Methods in Medical Imaging and Image Analysis, Principles of Ultrasound Modalities, World Scientific Publishing Co. Pte. Ltd. Chapter 1, PP 129-149, 2008 .

[3] Mittal, D., Kumar, V., Saxena, S.C., Khandelwal, N., Karla, N.: Enhancement of the ultrasound images by modified anisotropic diffusion method. Med. Biol. Eng. Comput. 48(12), 1281-1291 (2010). [4] William K Pratts, Digital Image Processing, Wiley, New York, 2006 PP 263-277.

[5] Loupas, T.: An adaptive weighted median filter for speckle suppression in medical ultrasonic images. IEEE Trans. Circuits Syst. 36 (1), 129-135 (1989)

[6] Zhang, F., Yoo, Y.M., Koh, L.M., Kim, Y.: Nonlinear diffusion in Laplacian pyramid domain for ultrasonic speckle reduction. IEEE Trans. Med. Imaging 26 (2), 200-211 (2007).

[7] Starck, J.L., Candes, E.J., Donoho, D.L.: The curvelet transform for image denoising. IEEE Trans. Image Process. 11(6), 670-684 (2002).

[8] Goel, P., Sinha, H.P., Singh, H.: Ultrasonic image denoising using multiscale ridgelet transform with hard and neighcoeff thresholding. Int. J. Comput. Commun. Technol. 2 (7), 65-70 (2011).

[9] Do, M., Vetterli, M.: The finite ridgelet transform for image representation. IEEE Trans. Image Process. 12 (1), 16-28 (2003).

[10] Donoho, D.L., Johnstone, I.M.: Adapting to unknown smoothness via wavelet shrinkage. J. Am. Stat. Assoc. 90, 1200-1224 (1995).

[11] A. Athanasiou, A. Tardivon, L. Ollivier, F. Thibault, C.E. Khoury, S. Neuenschwander, How to optimize breast ultrasound, Eur. J. Radiol. 69 (1) (2009) 6-13.

[12] I. Njeh, O.B Sassi, K Chtourou, A.B Hamida, Speckle noise reduction in breast ultrasound images: SMU (SRAD Median Unsharp) approach, in: Proceedings of the Eighth IEEE International Multi Conference on Systems, Signals and Devices, 2011, pp. 1-6.

[13] K.M. Prabusankarlal, P. Thirumoorthy, R. Manavalan, Computer aided breast cancer diagnosis techniques in ultrasound: a survey, J. Med. Imag. Health Inf. 4 (3) (2014) 331-349.

[14] Fabio Baselice, Giampaolo Ferraioli, Michele Ambrosanio, Vito Pascazio, Gilda Schirinzi, Enhanced Wiener filter for ultrasound image restoration, Computer Methods and Programs in Biomedicine 153 (2018) 71-81.

[15] Alex F. de Araujo, Christos E. Constantinou, João Manuel R.S. Tavares, Smoothing of ultrasound images using a new selective average filter, Expert Systems With Applications 60 (2016) 96-106.

[16] Fabio Baselice, Ultrasound Image Despeckling Based On Statistical Similarity, Ultrasound in Med. \& Biol., Vol. 43, No. 9, pp. 2065-2078, 2017. 
[17] P. Jidesh, B. Banothu, Image despeckling with non-local total bounded variation regularization, Computers and Electrical Engineering, 2017, http://dx.doi.org/10.1016/j.compeleceng.2017.09.013

[18] J. Zhang, G. Lin, L. Wu, Y. Cheng, Speckle filtering of medical ultrasonic images using wavelet and guided filter, Ultrasonics (2015), doi: http://dx.doi.org/10.1016/j.ultras.2015.10.005

[19] Sameera V., Mohd Sagheer, Sudhish N. George, Ultrasound image despeckling using low rank matrix approximation approach, Biomedical Signal Processing and Control 38 (2017) 236-249, http://dx.doi.org/10.1016/j.bspc.2017.06.011.

[20] Syed Gibran Javed, Abdul Majid, Yeon Soo Lee, Developing a bio-inspired multi-gene genetic programming based intelligent estimator to reduce speckle noise from ultrasound images, Multimed Tools Appl, 2017, DOI 10.1007/s11042-017-5139-2.

[21] Younes Farouj, Jean-Marc Freyermuth, Laurent Navarro, Marianne Clausel, and Philippe Delachartre, Hyperbolic Wavelet-Fisz Denoising for a Model Arising in Ultrasound Imaging, IEEE Transactions on Computational Imaging, Vol. 3, no. 1, January 2017.

[22] Ayesha Saadia, Adnan Rashdi, Fractional order integration and fuzzy logic based filter for denoising of echocardiographic image, Computer Methods and Programs in Biomedicine (2016), http://dx.doi.org/doi: 10.1016/j.cmpb.2016.09.006.

[23] P.V. Sudeep, P. Palanisamy, Jeny Rajan, Hediyeh Baradaran, Luca Saba, Ajay Gupta, Jasjit S. Suri, Speckle reduction in medical ultrasound images using an unbiased non-local means method, Biomedical Signal Processing and Control 28 (2016) 1-8, http://dx.doi.org/10.1016/j.bspc.2016.03.001.

[24] Ayesha Saadia, Adnan Rashdi, A Speckle Noise Removal Method, Circuits Syst em and Signal Processing, DOI 10.1007/s00034-017-0687-2.

[25] Jayachandran Jai Jaganath Babu, Gnanou Florence Sudha, Adaptive speckle reduction in ultrasound images using fuzzy logic on Coefficient of Variation, Biomedical Signal Processing and Control 23 (2016) 93-103. http://dx.doi.org/10.1016/j.bspc.2015.08.001

[26] Karamjeet Singh, Sukhjeet Kaur Ranade , Chandan Singh , A hybrid algorithm for speckle noise reduction of ultrasound images , Computer Methods and Programs in Biomedicine (2017), doi: 10.1016/j.cmpb.2017.06.009. 
TABLE: EVALUATION METRIC OF ALL THE STATED PAPERS

\begin{tabular}{|c|c|c|c|c|}
\hline Reference & Authors & Technique & Evaluation Metrics & Type of image used \\
\hline [14] & Fabio Baselice et. al. & Enhanced Wiener filter & ENL, USDSAI, N, $\beta, \sigma$. & simulated and real data sets \\
\hline$[15]$ & Alex F. de Araujo & Selective filter & PSNR, EPI, VIF, MSSIM and IQI & Synthetic images \\
\hline$[16]$ & Fabio Baselice & Non-local Means filter & ENL, USDSAI & simulated and real data sets \\
\hline [17] & P. Jidesh & non-local total bounded variation & PSNR, SSIM, $\lambda, \delta, \beta, \mathrm{g}$ & $\begin{array}{l}\text { ultrasound (US) and } \\
\text { synthetic aperture radar } \\
\text { (SAR) }\end{array}$ \\
\hline [18] & Ju Zhang & $\begin{array}{l}\text { improved wavelet filter and guided } \\
\text { filter }\end{array}$ & PSNR,SSIM, FoM Time(s) & $\begin{array}{l}\text { medical ultrasound images, } \\
\text { Synthetic image, Simulated } \\
\text { ultrasound image }\end{array}$ \\
\hline$[19]$ & Sameera V. et. al. & $\begin{array}{l}\text { low rank matrix approximation } \\
\text { approach }\end{array}$ & PSNR, MSE, EPI, SSIM. & $\begin{array}{l}\text { simulated and real } \\
\text { ultrasound data }\end{array}$ \\
\hline$[20]$ & Syed Gibran Javed et. al. & multi-gene genetic programming & $\begin{array}{l}\text { Fitness Criteria, Initial Population Size, } \\
\text { Max Generations, Selection Method, } \\
\text { Multi-Gene, Maximum No. of Genes, } \\
\text { Population Initialization Method, } \\
\text { Operator Probabilities, Survival Criterion }\end{array}$ & $\begin{array}{l}\text { Ultrasound images of } \\
\text { Breast Cyst, Kidney } \\
\text { Cancer, Liver, Liver Cyst, } \\
\text { and Fetal Head. }\end{array}$ \\
\hline$[21]$ & Younes Farouj et. al. & Hyperbolic Wavelet-Fisz & PSNR, Blocks SSIM, Kidney SSIM, & ultrasound images \\
\hline$[22]$ & Ayesha Saadia et. al. & $\begin{array}{l}\text { Fractional order integration and fuzzy } \\
\text { logic }\end{array}$ & $\rho, \beta, \Gamma, \mathrm{PSNR}, \mathrm{SSIM}$ & $\begin{array}{l}\text { standard test images } \\
\text { artificially corrupted with } \\
\text { speckle noise and real } \\
\text { ultrasound B-mode images } \\
\end{array}$ \\
\hline [23] & P.V. Sudeep et. al. & Unbiased non-local means method & PSNR, Pratt-FOM, MSSIM, BC & $\begin{array}{l}\text { phantom } \\
\text { images and real 2D } \\
\text { ultrasound datasets }\end{array}$ \\
\hline$[24]$ & Ayesha Saadia et. al. & $\begin{array}{l}\text { Fractional integral filter, average } \\
\text { adaptive filter }\end{array}$ & $\sigma_{n}, \mathrm{MSE}, \mathrm{PSNR}, \beta$, SSIM, $\rho$ & ultrasound images \\
\hline$[25]$ & $\begin{array}{l}\text { Jayachandran Jai } \\
\text { Jaganath Babu et. al. }\end{array}$ & $\begin{array}{l}\text { fuzzy logic on Coefficient of } \\
\text { Variation }\end{array}$ & $\beta, \mathrm{SNR}, \sigma n$ & $\begin{array}{l}\text { natural images, Field II } \\
\text { simulated images and real } \\
\text { ultrasound images, }\end{array}$ \\
\hline$[26]$ & Karamjeet Singh & $\begin{array}{l}\text { Guided filter, Guided Speckle } \\
\text { reducing Bilateral Filter (GSRBF), } \\
\text { Rotation Invariant Bilateral nonlocal }\end{array}$ & MSE, SNR, MSSIMM & $\begin{array}{l}\text { synthetic, simulated and } \\
\text { real ultrasound images }\end{array}$ \\
\hline
\end{tabular}


Mean Structural Similarity Index Matrix (MSSIM); Pratt's Figure of Merit (Pratt-FOM); Bhattacharya coefficient (BC) Speckle Suppression Index (SSI); Standard deviation of noise (n); Edge Preservation Index $\beta$

ENL Equivalent Number of Looks, Ultrasound Despeckling Assessment Index, $\mathbf{N}$ degree of smoothness.

edge preservation index (EPI) 